\title{
Crystal Shape Engineering
}

\author{
Michael A. Lovette, Andrea Robben Browning, Derek W. Griffin, \\ Jacob P. Sizemore, Ryan C. Snyder and Michael F. Doherty* \\ Department of Chemical Engineering \\ University of California \\ Santa Barbara, CA 93106-5080 USA
}

June 19, 2008

\begin{abstract}
In an industrial crystallization process, crystal shape strongly influences end-product quality and functionality as well as downstream processing. Additionally, nucleation events, solvent effects and polymorph selection play critical roles in both the design and operation of a crystallization plant and the patentability of the product and process. Therefore, investigation of these issues with respect to a priori prediction is and will continue to be an important avenue of research. In this review, we discuss the state-of-the-art in modeling crystallization processes over a range of length scales relevant to nucleation through process design. We also identify opportunities for continued research and specific areas where significant advancements are needed.
\end{abstract}

${ }^{*}$ To whom correspondence should be addressed. Phone: (805) 893-5309 e-mail: mfd@engineering.ucsb.edu 


\section{Introduction}

Crystallization from solution is a process used in the chemical industries for the preparation of many types of solids (e.g., pharmaceutical products, chemical intermediates, specialty chemicals, catalysts). Several key properties of the resultant materials originate from this process, including chemical purity and composition, internal structure (polymorphic state), size and shape distributions and defect density (crystallinity). Size and shape distributions impact various solid properties including end-use efficacy (e.g., bioavailability for pharmaceuticals, reactivity for catalytics ${ }^{1}$ ), flowability, wettability and adhesion. In turn, these properties impact down-stream processing efficiency (e.g., filtering/drying times and the possible need for milling), storage and handling.

The desired size and shape distributions of a crystalline product will vary depending on its application. For example, needle shaped (i.e., high-aspect ratio) crystals are well known to be difficult to process ${ }^{2}$ making them undesirable for pharmaceutical applications. However, needle shapes are key to several other applications (e.g., ZnO nanowires for solar cells), and in these cases crystal shapes are engineered to be as needlelike as possible.

Due to the broad impact of crystal size and shape distributions, general methodologies for the prediction, measurement and improvement of crystal size and shape are desired for product and process design. While these topics have been studied for over one hundred years, recent breakthroughs in experimental techniques, modeling and simulation have led to an improved understanding of crystal growth from solution. This article reviews some of these developments and presents a path for future progress.

The remainder of this article is organized as follows. It begins with a review of recent advances in the understanding of nucleation phenomena; highlighting experiments and simulations that have provided insight into the size, shape and structure of critical-sized nuclei. The shapes of larger crystals are discussed next. This discussion begins with a description of non-mechanistic models and then moves to a detailed discussion of crystal growth mechanisms. This is followed by a review of current modeling, experimental and simulation techniques for obtaining crystal shapes. Efforts in shape modification through the use of additives are described next, followed by methods to predict dynamics of crystal shapes evolving in time. Finally, crystal shape is discussed along with crystal size in the context of population balance models. 


\section{Nucleation and Polymorph Selection}

The role of nucleation in polymorph selection is of particular industrial interest. The polymorph (different crystal structures of the same molecule) obtained can have a large impact on the final utility (for example the Ritonavir case ${ }^{3}$ ) and patentability of a crystalline product. Different polymorphs can have different physical properties such as stability, hardness, color, solubility, dissolution rate and bioavailablity. ${ }^{4}$ Recent experiments have shown that the internal structure of a crystal may be set during nucleation. ${ }^{5}$ Furthermore, seeding experiments have shown that the seeded polymorph will not always appear in the final crystal. ${ }^{6}$ A method to predict the observed polymorph is an important goal in the study of nucleation. However, a more fundamental knowledge of nucleation must be achieved before predictive tools can be perfected.

Traditionally, Classical Nucleation Theory (CNT) has been used to describe gas, liquid and solid nucleation. ${ }^{7-12}$ Classical nucleation theory was laid out by Gibbs ${ }^{7}$ (page 257) in the 1870's, and has proven useful for understanding nucleation in a variety of systems, in particular gases and liquids. Nonetheless, Gibbs himself questioned its validity for solid nucleation.

Classical nucleation theory is based on the total change of the free energy of the system during a nucleation event. In a nucleation event, energy is decreased by the creation of the new lower energy phase (Equation 1, first term), and energy is increased by the formation of an interface between the two phases (Equation 1, second term). For a spherical nucleus the change in Gibbs free energy resulting from the creation of a nucleus with radius $r$ is given by

$$
\Delta G=-\frac{4}{3} \frac{\pi r^{3} \Delta \mu}{V_{M}}+4 \pi r^{2} \gamma
$$

where $V_{M}$ is the molar volume of the solid nucleus, $\gamma$ is the specific surface energy of the solidliquid interface assumed isotropic in this case (i.e., the same in all directions), $\Delta \mu$ is the chemical potential difference (on a per mole basis) between solute in solution and in the nucleus $(\Delta \mu>0$, for supersaturated solutions). The free energy surface, $\Delta G(r)$, resulting from Equation 1 has a single stationary point occurring at a maximum in $\Delta G$ (for anisotropic nuclei, this will occur at a saddle point as shown for 2D nucleation in Figure 8), yielding a critical-sized nucleus of radius $r_{c}$, given by

$$
r_{c}=\frac{2 \gamma V_{M}}{\Delta \mu}
$$


The change in $\Delta G$ of the system at this stationary point is given by

$$
\Delta G_{c}=\frac{16}{3} \frac{V_{M}^{2}}{\Delta \mu^{2}} \pi \gamma^{3}=\frac{4}{3} \pi r_{c}^{2} \gamma
$$

If a cluster is larger than this critical size it has a higher probability to grow than decay, below it has a higher probability to decay. This indicates that nucleation (as predicted by CNT) is a thermally activated process with a free energy barrier height of $\Delta G_{c}$, which therefore occurs at a rate given by

$$
I=\kappa_{3 D} \exp \left(-\Delta G_{c} / k_{B} T\right)
$$

where $I$ is the nucleation rate which has dimensions number of nuclei/(volume-time), $\kappa_{3 D}$ is a kinetic pre-factor, $k_{B}$ is and $T$ is temperature. ${ }^{13}$

As shown in Equation 2, the critical nucleus size is affected by surface energy, supersaturation and nucleus shape. As the supersaturation increases and the system moves away from equilibrium $(\Delta \mu \uparrow)$, the critical nucleus size will decrease. It is important to note that the shape and polymorph of the nucleus (the latter of which impacts $\gamma$ ) must be specified a priori in using CNT to determine $r_{c}$ and $\Delta G_{c}$.

It cannot be assumed that the surface energy of a critical nucleus is identical to that of the bulk solid, since critical-sized nuclei are small, $\mathcal{O}(1 \mathrm{~nm})$, and their structures may differ from the equilibrium bulk structures. ${ }^{14}$ This has been supported by recent simulations of $\mathrm{NaCl}^{15}$ and $\mathrm{SF}_{6} \cdot{ }^{16}$ However, the magnitude of the deviation in surface energy between critical-sized nuclei and bulk solids is not known. In addition to the differences in overall structure, the nucleus may have a different shape than the final crystal, revealing different faces and their different surface energies. Furthermore, the nucleus may even be spherical/elliptical in shape, in which case the concept of faces does not apply. Since critical nuclei are small, no direct experimental surface energy measurements on them have been made. This causes all theories and ideas about the correct nucleus surface energy to remain experimentally unverified. In addition to the question of surface energy, surface work terms, such as surface stress, may also need to be included for an accurate theory of solid nucleation. ${ }^{17}$ Surface stress, though unimportant in fluid nucleation, may become significant when the nucleus surface can experience strain.

In recent years, the theory of two step crystallization (also called two step nucleation) has attracted attention. In two step crystallization, nucleation proceeds through a dense liquid (amor- 
(a)

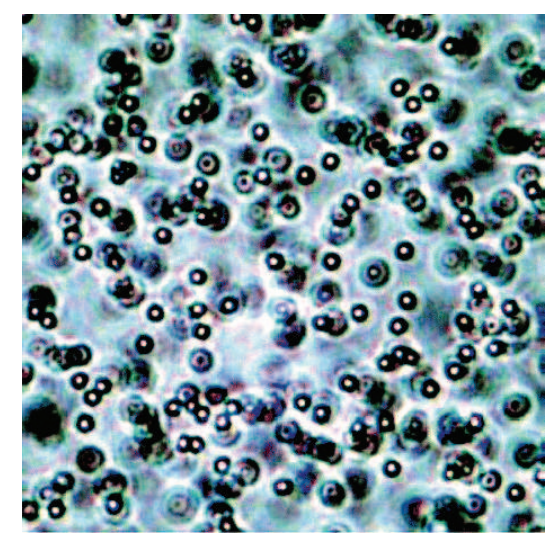

(b)

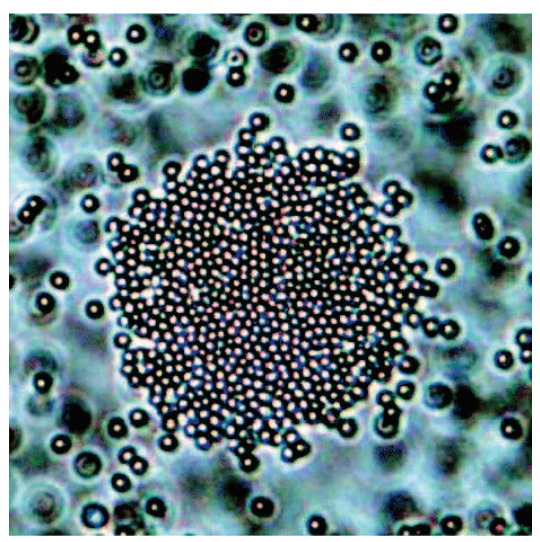

(c)

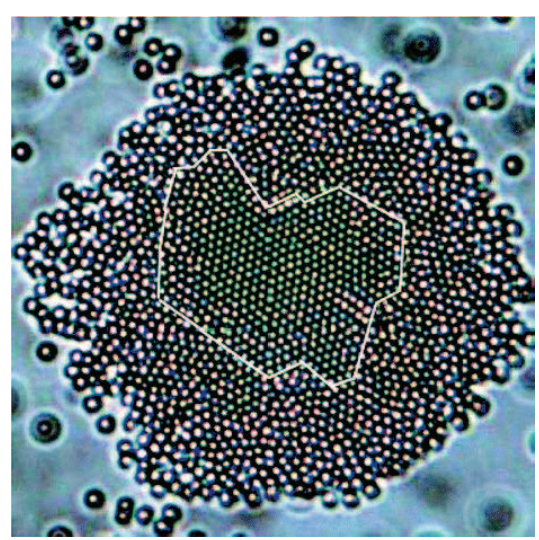

Figure 1: Two step nucleation of colloidal particles. Image (a) shows the initial liquid phase, (b) shows the dense liquid nucleus created first, followed by (c) the crystalline nucleus forming in the dense liquid. ${ }^{25}$

phous) step before ordering into the growth structure. ${ }^{18}$ This theory is supported by both simulations ${ }^{19-22}$ and experiments. ${ }^{23-25}$ Two step crystallization has been experimentally observed in protein and colloidal systems and in simulations of $\mathrm{MnO}_{2}$. The two step progression from liquid to crystalline nuclei observed in colloid experiments can be seen in Figure 1. Since CNT assumes a single step, the evidence supporting two step crystallization raises the question of how to include a multi-step process in nucleation theory. ${ }^{23}$

Experimental studies and simulations are being actively pursued in order to enable methods of nucleus polymorph and shape prediction. However, nuclei are difficult to observe experimentally as they are often indistinguishable from their parent solution due to their small size $(\mathcal{O}(100$ particles $))$ and low concentration. Frenkel and coworkers ${ }^{26}$ estimated that for melt $\mathrm{NaCl}$ crystallization, a single nucleus would form in a droplet of diameter $\mathcal{O}(3 \mu \mathrm{m})$ in $\mathcal{O}(10 \mathrm{~s})$ which highlights the rarity of nucleation in terms of experimental observation. Designing experiments to ensure that nuclei are observed before significant growth has occurred remains a challenge.

These challenges have been overcome in large particle experiments where nucleation has been directly observed. ${ }^{5,27}$ Colloids and globular proteins are large enough and nucleate slowly enough for nucleation to be observed using techniques such as optical and atomic force microscopy (AFM). The colloidal and protein nucleation experiments performed by Gasser et al. ${ }^{27}$ and Yau and Vekilov, ${ }^{5}$ 
(a)

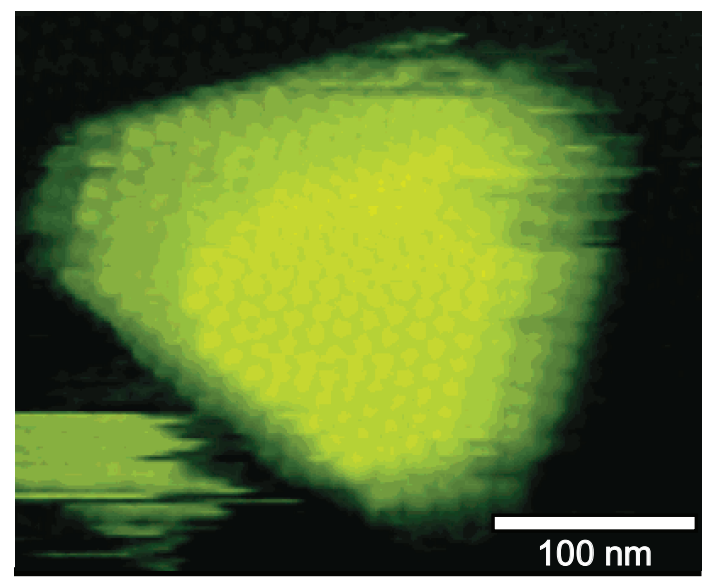

(b)

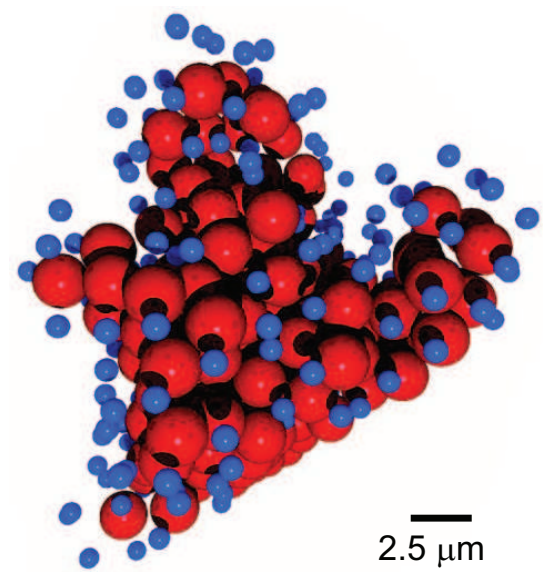

Figure 2: Atomic Force Microscopy (AFM) image (a) of a critical nucleus of apoferritin molecules with molecules in nucleus shown in green and (b) a critical nucleus of poly(methyl methacrylate) colloids with particles in the nucleus shown in red and particles with only one bond to the nucleus shown in blue with reduced size for clarity. Images (a) and (b) were adapted from Yau and Vekilov ${ }^{5}$ and Gasser et al., ${ }^{27}$ respectively.

respectively, found critical nuclei with 25-100 particles and non-spherical shapes, as shown in Figure 2. In both cases the internal structure of the critical nuclei corresponded to the thermodynamically stable polymorphs. ${ }^{4}$ Small-angle neutron scattering techniques have also been used to observe nucleation of polymer blends. ${ }^{28}$ The results of these experiments have provided new insights into the nucleation process.

For small molecules, direct measurement and observation of nuclei is currently impossible. Though limited by the small length and short time scales, indirect measurements of nucleation can be made. ${ }^{29-32}$ Indirect methods include the observation of crystal particles after growth to a larger size and characterization of the final crystal structure, with attempts made to keep the nucleation rate low after initial nuclei formation. These methods can measure average nucleation rates, induction time and the effects of experimental conditions on crystal structures.

The development of new computational techniques coupled with increases in computing power have recently allowed for nucleation phenomena to be simulated. To date, most nucleation simulations have been performed using model particles with simple pair-additive potentials. ${ }^{33-39}$ The model particle studies performed by Browning et al. ${ }^{39}$ found critical nuclei similar to the protein 

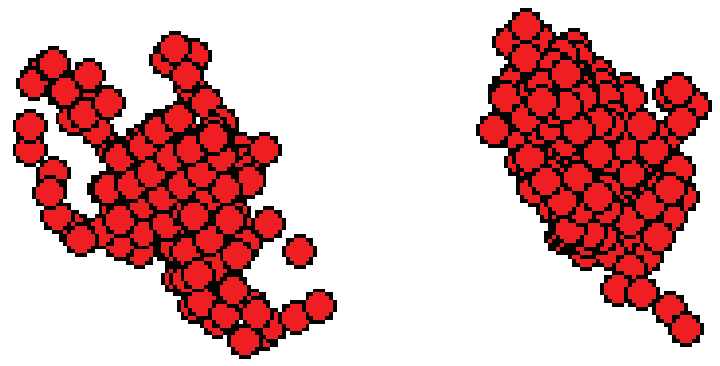

Figure 3: Near critical size nuclei found in model particle simulations performed by Browning et al.. ${ }^{39}$

and colloid experiments with sizes of $\sim 100$ particles and non-spherical shape, shown in Figure 3. In these simulations, the majority of the critical nucleus's structure corresponded to that of the stable polymorph; however, some percentage of the metastable polymorph was also found.

Nucleation simulations using interaction potentials based on small molecules have also been performed. Simulations of amorphous silicon found critical nuclei containing 30-50 atoms ( 1.2 $\mathrm{nm}$ in diameter) with nearly spherical shape. ${ }^{40}$ critical-sized nuclei of $\mathrm{N}_{2}$ were found to contain approximately 300 molecules. ${ }^{41}$ Faceted critical nuclei of similar size have been observed in melt nucleation simulations of $\mathrm{NaCl}^{26}$ and $\mathrm{SF}_{6} \cdot{ }^{16}$ A summary of selected critical nucleus sizes and shapes is reported in Table 1.

Due to the time and size scale difficulties of simulating nucleation, pre-critical aggregates have also been studied. Simulations of $\mathrm{NaCl}$ in water showed that the centers of stable aggregates had non-hydrated sodium ions. ${ }^{42}$ Acetic acid aggregation in carbon tetrachloride solution was found to proceed through dimer formation to oligomers to hydrogen bonded micelles. ${ }^{43}$ New simulation techniques such as reaction coordinate calculation and transition path sampling ${ }^{44,45}$ may soon allow for a more complete study of small molecule nucleation from solution.

The nucleation stage, wherein pre and post critical-sized clusters $(\sim(100-10,000)$ molecules and $\sim 10 \mathrm{~nm}$ in diameter) grow by aggregation, is now being actively explored using molecular dynamics (MD), Monte Carlo (MC) and other simulation techniques. However, after a crystallite has grown to a microscopic size on the order of millions of particles and $\sim 1000 \mathrm{~nm}$, other methods of growth have been observed. These methods are discussed in the remainder of this article. What occurs during the transition between the nucleation and macroscopic growth stages is presently unknown. 
Table 1: Summary of selected critical nucleus sizes determined by experiment (apoferritin) and simulations ( $\mathrm{SF}_{6}, \mathrm{NaCl}$, and Lennard-Jones particles). The general agreement between the different systems and the decrease in critical nucleus size with increasing driving force can be seen.

\begin{tabular}{|c|c|c|c|}
\hline System & Driving Force & $\begin{array}{c}\text { Critical Nucleus Size } \\
\text { (\# particles or molecules) }\end{array}$ & Shape \\
\hline \multirow[t]{3}{*}{ Apoferritin $^{5}$} & 43.7 & 4 & quasi-planar \\
\hline & 3.95 & 25 & (faceted) \\
\hline & 2.00 & 60 & \\
\hline \multirow[t]{3}{*}{$\mathrm{SF}_{6}{ }^{16}$} & 0.20 & 40 & anisotropic \\
\hline & 0.13 & 120 & (faceted) \\
\hline & 0.086 & 266 & \\
\hline \multirow[t]{2}{*}{$\mathrm{NaCl}^{26}$} & 0.25 & 120 & cubic \\
\hline & 0.22 & 150 & (faceted) \\
\hline Lennard-Jones 37 & 0.2 & 240 & ellipsoidal \\
\hline
\end{tabular}

where Driving Force is $C / C_{e q}-1$ for apoferritin and $1-T / T_{\text {melt }}$ for remaining systems 
The methods that have been used to study nucleation are not sufficient to study this regime and therefore new methods must be developed, which will surely give rise to a lively and dynamic research area in the next decade.

\section{Equilibrium and Growth Shapes}

Equilibrium criteria for the interfaces dividing solids and fluid phases were developed by Gibbs. ${ }^{7}$ Wulff developed a geometric approach for determining the shapes of faceted crystals (at constant temperature and pressure) with anisotropic surface free energies conforming to these criteria, known as the Wulff construction. ${ }^{46,47}$ The work of formation of a solid polyhedron with volume $V$ from a fluid phase is given by the Gibbs-Thomson formula

$$
\Delta G=-\frac{V \Delta \mu}{V_{M}}+\sum_{i} \gamma_{i} A_{i}
$$

where $\gamma_{i}$ and $A_{i}$ are the surface free energy and area of the $i^{\text {th }}$ facet, respectively. ${ }^{48}$ The equilibrium shape occurs when $\sum_{i} \gamma_{i} A_{i}$ is a minimum for fixed crystal volume, $V$, and can be determined from

$$
\sum_{i} \gamma_{i} \mathrm{~d} A_{i}=0
$$

The Wulff construction (which yields the equilibrium shape) is obtained by connecting the exterior end points of vectors, with origins located at the center of the crystal, to planes with angles perpendicular to them and magnitudes, $H_{i}$, proportional to their corresponding surface free energies; resulting in ${ }^{48,49}$

$$
\frac{\gamma_{1}}{H_{1}}=\frac{\gamma_{2}}{H_{2}}=\cdots=\frac{\gamma_{i}}{H_{i}}
$$

While Gibbs was the first to develop the criteria for equilibrium crystal shapes; he recognized, as footnoted in his collected works (page 325), that crystal shapes are usually determined by kinetics rather than by thermodynamics alone. ${ }^{7}$

"On the whole it seems not improbable that the form of very minute crystals in equilibrium with solvents is principally determined [...] by the condition that $\sum_{i} \gamma_{i} A_{i}$ shall be a minimum for the volume of the crystal, [...] but as they grow larger (in a solvent no more supersaturated than is necessary to make them grow at all), the deposition of new matter on the different surfaces will be determined more by the 
nature (orientation) of the surfaces and less by their size and relations to the surrounding surfaces. As a final result, a large crystal, thus formed, will generally be bounded by those surfaces alone on which the deposit of new matter takes place least readily (i.e., the slow-growing faces) [....] But the relative development of the different kinds of sides $[\ldots]$ will not be such as to make $\sum_{i} \gamma_{i} A_{i}$ a minimum." $\dagger$

In contrast to fluids, the resistance to deformations ubiquitous with solids requires that shape changes occur through the deposition and removal of material at crystal faces. Therefore, the shapes of growing crystals will be determined by the net rate of material incorporation into individual surfaces rather than by their equilibrium shapes. If the net rate of material incorporation is constant on each face, a crystal will evolve towards its steady state growth shape, at which point growth proceeds in a self-similar manner. This steady state growth shape is generally not equivalent to the equilibrium shape.

Analogous to the Wulff construction for predicting equilibrium shapes; the Frank-Chernov condition $^{50,51}$ can be used to predict steady state growth shapes for faceted crystals, provided the crystallographic data and growth rates of the faces are known. Thus, the remaining sections of this article (with the exception of $\S 8$ ) will focus on the development of theories, simulations and experiments that have contributed to the elucidation of crystal growth kinetics. The Frank-Chernov condition has the same form as the Wulff construction, with the exception that the shape is determined from the perpendicular growth rates of faces and not surface energies,

$$
\frac{G_{1}}{H_{1}}=\frac{G_{2}}{H_{2}}=\cdots=\frac{G_{i}}{H_{i}}
$$

where $G_{i}$ is the perpendicular growth of the $i^{\text {th }}$ face. Additionally, methods to predict shape evolution in both growth ${ }^{52,53}$ (discussed in $\S 7$ ) and dissolution ${ }^{53}$ have been developed.

The first approaches for predicting growth shapes were based solely on the structure and interactions within the crystal. Bravais ${ }^{54}$ proposed a quantitative relationship for predicting crystal shape based on crystal structure, which gained validity from the observations of Friedel. ${ }^{55}$ Bravais' relationship is

$$
G_{h k l} \propto \frac{1}{d_{h k l}}
$$

\footnotetext{
${ }^{\dagger}$ The words in italics are ours and [...] indicate words that we left out for brevity and/or clarity.
} 
where $G_{h k l}$ and $d_{h k l}$ are the perpendicular growth rate and interplanar spacing, respectively, of the face specified by the index $h k l$. Donnay and Harker ${ }^{56}$ modified this approach by including reductions in $d_{h k l}$ due to extinction conditions. These conditions are the same as those that cause extinction in x-ray diffraction patterns. This model (referred to as the BFDH model) represents the most easily implemented method for shape prediction, since it requires only a knowledge of the crystallography of the crystal.

Later, the attachment energy model, developed by Hartman and Perdock, ${ }^{57,58}$ took into account the energetics of crystal interactions in addition to the crystal geometry. They assumed that the time needed for the formation of a bond decreases with increasing bond energy. Defining the attachment energy, $E_{h k l}^{a t t}$, as "the bond energy released when one building unit is attached to the surface of a crystal face," this assumption results in the perpendicular growth rates of a crystal face increasing with increased attachment energy, yielding the relationship

$$
E_{m}^{a t t}>E_{n}^{a t t} \Rightarrow G_{m}>G_{n}
$$

where $m$ and $n$ are the indices describing two different faces. For a crystal face the attachment energy can be determined from

$$
E^{l a t t}=E_{h k l}^{\text {slice }}+E_{h k l}^{a t t}
$$

where $E^{\text {latt }}$ is the total energy of intermolecular interactions per mole in a crystal and $E_{h k l}^{\text {slice }}$ is the energy of the intermolecular interactions contained within a layer of thickness $d_{h k l}{ }^{59}$ To obtain the perpendicular growth rates from Equation 10, the assumption of linearity is often made, ${ }^{60}$ i.e.,

$$
G_{h k l} \propto E_{h k l}^{a t t}
$$

Since its inception, the attachment energy model has been further modified ${ }^{61}$ and is used extensively to predict crystal shape. In recent years, however, there has been a growing trend away from the BFDH and attachment energy models towards higher fidelity mechanistic models. These models appear to be more accurate and versatile.

\section{Growth Processes}

While the BFDH and attachment energy models can provide accurate predictions for vapor grown crystals, they account only for the influence of solid-side energetics on crystal shape. In doing 


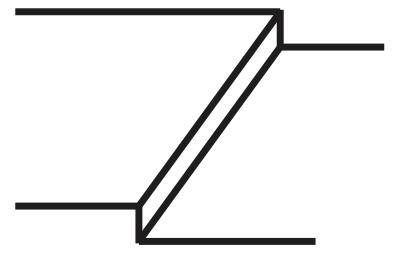

(a)

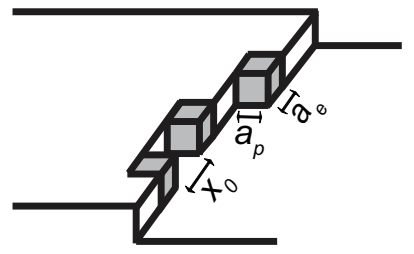

(b)

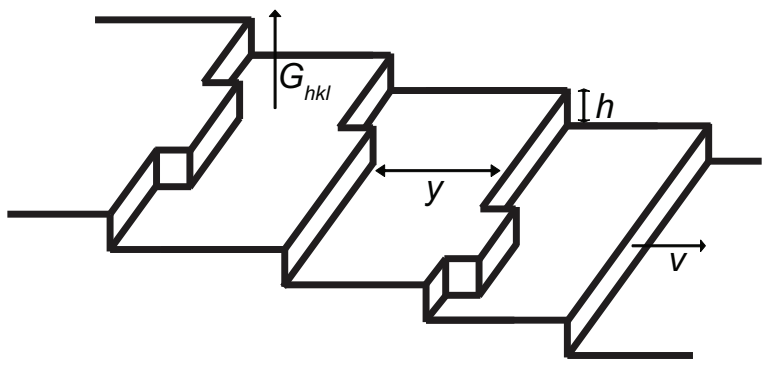

(c)

Figure 4: Schematic of step edges at $0{ }^{\circ} \mathrm{K}$ (a) and above $0{ }^{\circ} \mathrm{K}(\mathrm{b}$ and $\mathrm{c}$ ). The grey squares in (b) represent kink sites separated by an average distance of $x_{0}$. Image (c) is a schematic of layered growth of the $\{\mathrm{hkl}\}$ face growing at a perpendicular growth rate, $G_{h k l}$, through the lateral spreading of steps separated by an interstep distance, $y$, with a height, $h$, at a step velocity of $v$.

so, they ignore the influence of external factors on crystal growth such as solvent, additives and supersaturation, which can impact their accuracy to varying degrees for solution crystallization. An understanding of growth processes, namely the creation of kink sites and transport of material to growing faces, is required to develop models that can account for these factors.

Kink sites are the positions on a crystal where molecules can most readily incorporate into or detach from the lattice and appear as exposed trihedral corners (shown as grey squares in Figure $4 \mathrm{~b})$. At a classical kink site, the adjacent molecule in the crystal is bound by half of the possible intermolecular interactions for a molecule incorporated into the lattice, ${ }^{62,63}$ thus their description as "half crystal positions". The method by which kink sites are created on a crystal face depends on solid-state factors and the state of the growth environment.

During growth of a crystal face from solution the following processes occur in series: (1) solute molecules are transported from the bulk solution towards the face by convection and diffusion (2) solute molecules and kink sites shed their surrounding solvent molecules (desolvate) and solute molecules incorporate into kink sites and (3) the latent heat of crystallization is released and 


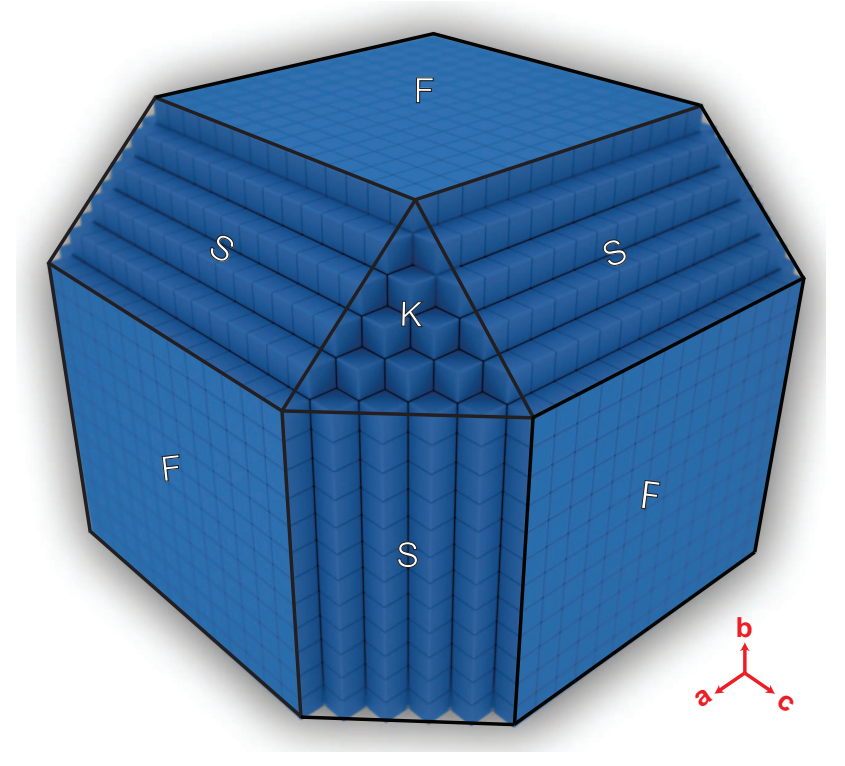

Figure 5: F, S and K faces for a crystal with 3 PBCs, shown in red.

transported to the crystal and solution. For clarity: process 1 is hereafter referred to as bulk transport, process 2 is referred to as surface integration and process 3 is referred to as heat transport. The growth rate of a crystal face is limited by the slowest of these processes. ${ }^{64,65}$ Kink sites are created at a certain density on the face by processes occurring simultaneously with processes (1)-(3). The density of kink sites is included as a contribution to the rate of surface integration.

One important description of the solid-state for a molecular crystal is its (non-covalent) bond structure, which can be discussed using periodic bond chain (PBC) theory, proposed by Hartman and Perdok. ${ }^{57}$ PBCs have been defined as a set of connections between growth units at their centers of mass following only "strong" interactions with an overall periodicity $[u v w]=u \mathbf{a}+v \mathbf{b}+w \mathbf{c}$, $(u, v, w \in Z)$ where $\mathbf{a}, \mathbf{b}$ and $\mathbf{c}$ are the primitive lattice vectors co-incident with the unit cell directions and $Z$ consists of all positive and negative integers. ${ }^{60}$ Using PBC theory, faces can be classified based on the number of coplanar PBCs they contain within a slice of thickness $d_{h k l}$. If a face contains at least two PBCs, it is stable and macroscopically flat (F face); if it has only one PBC then it is stepped ( $\mathrm{S}$ face) where each step can be readily kinked; if it has none, then it is completely kinked and rough (K face). These faces are shown in Figure 5.

The mechanism by which a face grows can also depend on the state of the external environment, specifically the supersaturation and temperature at the surface. In general, a face will grow by 


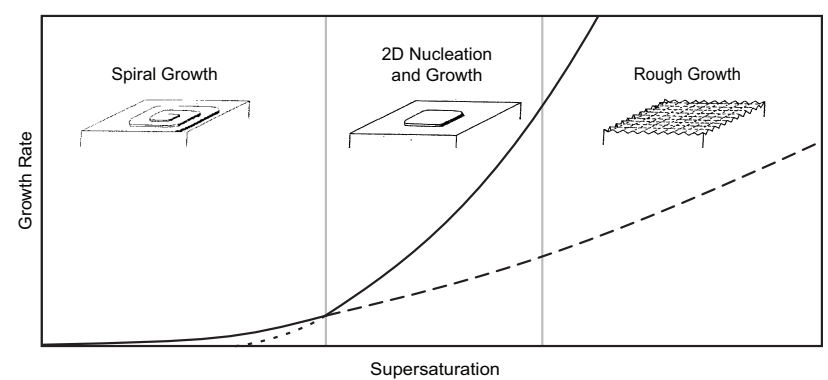

Figure 6: Growth mechanisms for a flat (F) face as a function of supersaturation. The solid line is the growth rate. The short dashed lines are the growth rates if $2 \mathrm{D}$ nucleation is continued to be dominant below its applicable supersaturation range. The long dashed line is the rate if spiral growth was the persistent mechanism above its applicable supersaturation range.

whichever process (or combination of processes) provide the fastest growth rate for a given state of the growth environment. While $\mathrm{S}$ and $\mathrm{K}$ faces grow by rough growth (described in §4.1) at all states of the growth environment, $\mathrm{F}$ faces grow by a layered growth mechanism (described in $\S 4.2$ ) until the state of the growth environment is above a roughening transition (described in $§ 4.1$ ). This is shown schematically for $\mathrm{F}$ faces in Figure 6, which assumes that the growth rate is limited by the rate of surface integration for all growth mechanisms. The transition between growth mechanisms has been illustrated experimentally using in situ atomic force microscopy (AFM) by Land and De Yoreo. ${ }^{66}$

\subsection{Rough Growth}

Some faces, such as the $\{111\}$ faces of a Kossel crystal (the classic example of a $\mathrm{K}$ face, shown in Figure 5) are composed almost entirely of kink sites. On these faces, molecules can incorporate into the crystal regardless of their position and growth is typically limited by the transport of solute molecules to the surface, resulting in growth rates given by:

$$
G_{B T} \sim V_{M}\left(C_{\infty}-C_{e q}\right) \frac{\mathcal{D}}{\delta_{d}}
$$

where $G_{B T}$ is growth rate limited by the bulk transport of material to the surface, $C_{\infty}$ is the bulk concentration of the solute in solution, $C_{e q}$ is the equilibrium saturation concentration of the solute in solution, $\mathcal{D}$ is the diffusion coefficient of the solute in the solvent and $\delta_{d}$ is the thickness of the depletion boundary layer wherein the concentration deviates from the bulk. Equation 13 (derived in 
the Supplementary Material) assumes the solute concentration at the crystal face is maintained at $C_{e q}$. Typical values for molecular organic crystals grown from solution in an industrial crystallizer are $V_{M}\left(C_{\infty}-C_{e q}\right) \approx 0.005, \mathcal{D} \approx 10^{-5} \mathrm{~cm}^{2} / \mathrm{s}$ and $\delta_{d} \approx \mathcal{O}(1 \mu \mathrm{m})$, result in $G_{B T} \approx \mathcal{O}(5 \mu \mathrm{m} / \mathrm{s})$ which is $\sim(10-100)$ times larger than typical growth rates for faces growing by a layered growth mechanism. As a result of this large growth rate, rough faces are typically short lived in the evolution of a crystal's shape as they grow into edges or vertices. ${ }^{52}$ Needle shaped crystals are an industrially-important exception; as rough faces are present on and responsible for the highlyelongated steady state shape of needles.

F-faces will also grow by rough growth at supersaturations or temperatures beyond their kinetic and thermodynamic roughening transitions, respectively. These roughening transitions have been extensively studied and the reader is referred to Chernov ${ }^{67}$ for further discussion.

\subsection{Layered Growth}

For $\mathrm{F}$ faces below their roughening transitions, kink sites are found along edges that result from either 2D nuclei forming or dislocations emerging on the surface. As growth units incorporate into these kink sites the edges flow outwards from their sources; forming complete layers of the crystal face. In a supersaturated solution, this process repeats, resulting in the perpendicular growth of the face. Layered growth is a slower process than rough growth since the density of kink sites is much lower on an $\mathrm{F}$ face than on a rough face.

According to theory developed by Frenkel ${ }^{68}$ and extended by Burton et al., ${ }^{69}$ at $0{ }^{\circ} \mathrm{K}$ steps will be as straight as geometrically possible; however at any higher temperature they will contain kink sites (Figure 4a and b). If the kink sites along an edge are formed by thermal fluctuations (in contrast to one dimensional nucleation, for more on this subject the reader is referred to Zhang and Nancollas ${ }^{70}$ and Cuppen et al. ${ }^{71,72}$ ) and are located in their most probable configuration, they will be Boltzmann distributed along the steps with the distance between kinks, $x_{0}$, equal to

$$
x_{0}=a_{e}\left(1+1 / 2 \exp \left(\phi_{k i n k} / k_{B} T\right)\right)
$$

where $a_{e}$ is the lattice spacing in the direction along the edge (Figure $4 \mathrm{~b}$ ) and $\phi_{k i n k}$ is the energy (positive) required to form a kink. ${ }^{67}$ The energy to form a kink includes both solid-solid and solid-solvent interactions. 
Layered growth occurs by either 2D nucleation and growth or spiral growth, depending on the method of edge generation. In 2D nucleation and growth, 2D nuclei forming at the surface provide edges; whereas, in spiral growth, screw dislocations at the surface are constant sources of edges.

\subsubsection{Two-Dimensional Nucleation and Growth}

Crystal faces grow by the formation of critical-sized 2D nuclei in the absence of surface terminating screw dislocations or at supersaturations above which growth by $2 \mathrm{D}$ nuclei becomes faster than spirals (a method for determining this point is described in §5.1.1). These nuclei form when individual growth units (e.g., atoms, molecules, dimers) adsorb onto the crystal face, diffuse and agglomerate. After a nucleus has grown larger than its critical size, it becomes thermodynamically favorable for growth units to attach themselves to this nucleus. In a supersaturated solution, a larger than critical-sized 2D nucleus will spread laterally across the face until it reaches a boundary, which can be the edge of the crystal, the front of the layer beneath it or a growth front from another nucleus (or spiral).

\subsubsection{Spiral Growth}

In the presence of screw dislocations, crystal faces grow by the spiral growth mechanism at supersaturations lower than the value where $2 \mathrm{D}$ nucleation and growth is the dominant mechanism. In spiral growth, screw dislocations terminating on a crystal face act as continuous sources of edges (which will also be called steps in the discussion of this mechanism). At each dislocation an edge is exposed to the solution. If its length is greater than a critical value, $l_{c}$, and the solution is supersaturated at the surface, there will be a net incorporation of growth units into kink sites distributed along the edge. This incorporation causes the migration of the initial step in its outward normal direction. As the step moves it exposes another edge to the solution. This new step is pinned at the point where the dislocation emerged on the surface. The angle between steps is determined by the bond structure of the face. Once this newly exposed step has reached its critical length; it grows in its outward normal direction and the process repeats forming a spiral. The number of sides contained in the spiral is determined by the bond structure of the face and the velocities and critical lengths of the spiral sides. In a supersaturated solution the steps will continue to flow outwards until they encounter a boundary, which can be a step from another spiral or 2D nucleus, 
(a)

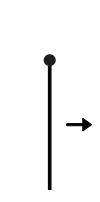

(1)

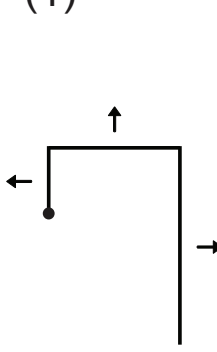

(4)

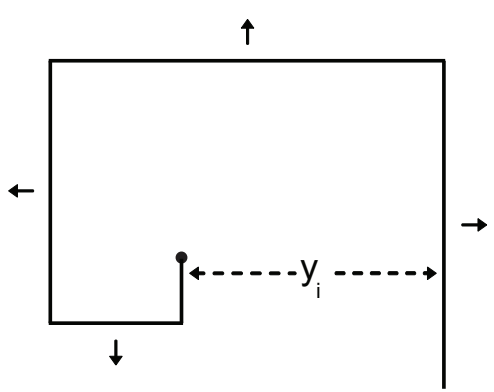

(6) (b)

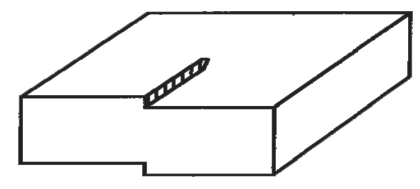

(1)

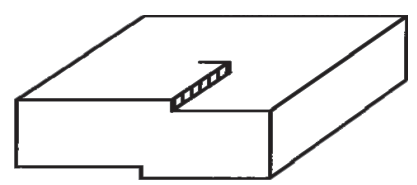

(3)

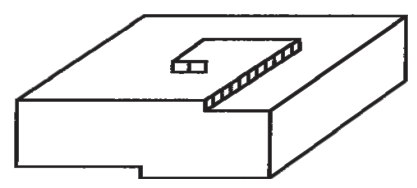

(5)

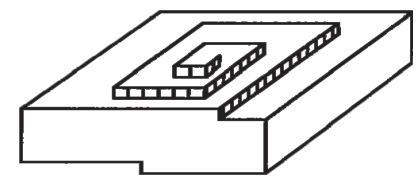

(6)

Figure 7: Top (a) and perspective (b) view schematics of the first revolution of a square spiral. The steps on this spiral obey the conditions: (i) $v_{i}=0$ for $l<l_{c}$ and $v_{i}=v_{\infty, i}$ for $l>l_{c}$, (ii) $v_{i}=v_{j}$ and (iii) $l_{c, i}=l_{c, j}$, resulting in interstep distances $y_{i}=4 l_{c}$.

the step located a layer below it or the edge of the face; and new steps will continually be generated at the dislocation. The process of spiral growth is illustrated in Figure 7.

\section{Obtaining Shapes from Mechanistic Models, Simulations and Experiments}

Crystal shape engineering, which can be defined as the ability to design processes through the modification of the crystallization environment to obtain desired crystal shapes, has benefited from the development of mechanistic models, computer simulations and experiments investigating 
crystal growth processes. These approaches, which have been used to obtain/predict crystal growth kinetics, have relied on each other in their development and will continue to do so.

\subsection{Mechanistic Crystal Growth Models}

Mechanistic growth models predict growth rates for crystal faces by accounting for surface integration kinetics. These models are useful for regimes where growth is limited by (1) surface integration alone, or by (2) both bulk transport and surface integration. For most molecular organic crystals grown from solution in an industrial crystallizer, this regime encompasses the states of the growth environment for which $\mathrm{F}$ faces grow by spirals, as will be shown in $§ 5.1 .2$. However, when growth is limited by bulk transport, the growth rate of the face is given by Equation 13 regardless of the growth mechanism. Mechanistic growth models are attractive for future development since they provide the ability to account for the influence of surface structure and external conditions (supersaturation, solvent, additives, etc.) on crystal growth rates in a physically realistic manner.

\subsubsection{Modeling Two-dimensional Nucleation and Growth}

Two-dimensional nucleation and growth models were the first mechanistic models used to describe crystal growth. ${ }^{62,63,73}$ The principle thesis of these models is that otherwise perfectly flat crystal faces grow by the formation of critical-sized 2D nuclei and their subsequent lateral growth. The growth rate of a crystal face growing by this mechanism is dependent upon the size of nuclei formed and their rates of formation and lateral spreading.

Classical nucleation theory has been used elsewhere to derive the change in free energy associated with the creation of critical-sized 2D nuclei of different shapes. ${ }^{67,69,74,75} \mathrm{~A}$ critical-sized 2D nucleus is an entity that exists in an unstable equilibrium with the system, occurring at a maximum or saddle point on the free energy landscape. As with 3D nucleation, growth of sub-critical-sized 2D nuclei occurs at an energetic expense while decay is an energetically favorable process. Conversely, the growth of larger than critically-sized 2D nuclei is energetically favorable, whilst their decay occurs at an energetic expense.

When the surface energy is isotropic on a face, disc-shaped nuclei are expected to form. The expressions for $\Delta G, r_{c}$ and $\Delta G_{c}$ are similar to Equations 1-3, except appropriately modified for 2dimensions. ${ }^{75}$ Interfacial energies along different directions on the faces of most molecular crystals 
are anisotropic, resulting in the formation of non-circular 2D nuclei. A parallelogram shaped 2D nucleus with anisotropic interfacial energies, $\gamma_{e d g e, 1}=\gamma_{e d g e, 3} \neq \gamma_{e d g e, 2}=\gamma_{e d g e, 4}$, is shown in Figure 8 a. For this case, $\Delta G$ is given by

$$
\Delta G=h\left(2\left(l_{1} \gamma_{e d g e, 1}+l_{2} \gamma_{e d g e, 2}\right)-\frac{\left(l_{1} l_{2}\right) \sin (\xi)}{V_{M}} \Delta \mu\right)
$$

where $\xi$ is the angle between sides 1 and 2 , and $l_{1}$ and $l_{2}$ are the lengths of sides 1 and 2 , respectively. In this case, the critical-sized nucleus occurs at a saddle point in the free energy landscape, shown in Figure $8 \mathrm{~b}$ and the critical lengths are given by

$$
l_{1, c}=\frac{2 V_{M} \gamma_{e d g e, 2}}{\sin (\xi) \Delta \mu}
$$

and

$$
l_{2, c}=\frac{2 V_{M} \gamma_{e d g e, 1}}{\sin (\xi) \Delta \mu}
$$

The shape of the critical nucleus is equal to the equilibrium shape, as it agrees with the Wulff construction (i.e., $l_{1, c} \gamma_{e d g e, 1}=l_{2, c} \gamma_{e d g e, 2}$ or $\frac{\gamma_{e d g e, 1}}{H_{1}}=\frac{\gamma_{e d g e, 2}}{H_{2}}$ where $H_{1}=\frac{l_{2, c}}{2} \sin (\xi)$ and $H_{2}=$ $\left.\frac{l_{1, c}}{2} \sin (\xi)\right)$. The resulting free energy of formation of the critical-sized nucleus is given by

$$
\Delta G_{c}=\frac{4 V_{M} h}{\sin (\xi) \Delta \mu} \gamma_{e d g e, 1} \gamma_{e d g e, 2}
$$

As with 3D nucleation (treated in $\S 2$ ), 2D nucleation is a thermally activated process with a free energy barrier height of $\Delta G_{c}$. Thus, nucleation rates, $I$, in units of nuclei/(area time) will be given by

$$
I=\kappa_{2 D} \exp \left(-\Delta G_{c} / k_{B} T\right)
$$

where $\kappa_{2 D}$ is a kinetic pre-factor. Ohara and Reid ${ }^{74}$ determined $\kappa_{2 D}$, obtaining

$$
I=\frac{2 \bar{v} n_{1}^{2}}{\pi}\left(\frac{V_{M} \sigma}{N_{A} h}\right)^{1 / 2} \exp \left(-\Delta G_{c} / k_{B} T\right)
$$

where $\bar{v}$ is the speed of a molecule striking a nucleus, $n_{1}$ is the concentration of unbound growth units on the surface, $\sigma \equiv \Delta \mu / R T$ and $N_{A}$ is Avogadro's number.

The ratio of the rates of lateral spreading to the formation of critical-sized nuclei determines the specific $2 \mathrm{D}$ nucleation and growth model that can be applied to characterize the growth of a crystal face. At relatively slow though non-negligible rates of lateral spreading, the birth and spread model can be applied. The assumptions used in this model are: (1) no intergrowth between 
(a)

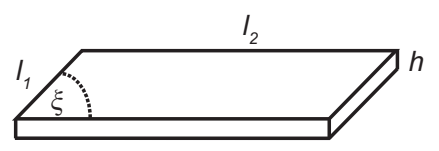

(b)

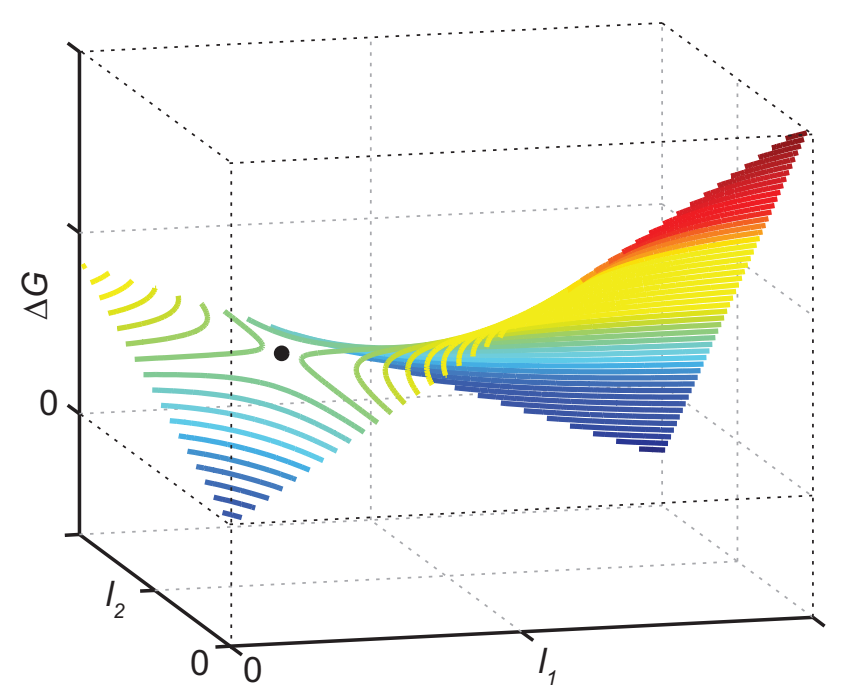

Figure 8: Geometry (a) and Gibbs free energy surface (b) for an anisotropic parallelogram-shaped 2D nucleus. The lines are contours or equal $\Delta G$ and the dot is the position of a critical-sized 2D nucleus, which occurs at a saddle point in the surface. 
Table 2: Edge energies and minimum values of $\alpha$ for observed growth by 2D nucleation for various compounds, adapted from Land and De Yoreo ${ }^{66}$ and De Yoreo and Vekilov. ${ }^{77}$

\begin{tabular}{lcccccc}
\hline Molecule & $\begin{array}{c}\text { Atoms per } \\
\text { Molecule }\end{array}$ & $\begin{array}{c}h \\
(\mathrm{~nm})\end{array}$ & $\begin{array}{c}\gamma_{\text {edge }} \\
\left(\mathrm{erg} / \mathrm{cm}^{2}\right)\end{array}$ & $\begin{array}{c}\gamma_{\text {edge }} a h \\
(\mathrm{erg})\end{array}$ & $\begin{array}{c}\alpha \text { for } \\
2 \mathrm{D} \text { nucleation }\end{array}$ & $N_{c}$ \\
\hline $\mathrm{CaCO}_{3}$ & 5 & 0.31 & 675 & $1.3 \times 10^{-12}$ & 20.1 & $<600$ \\
$\mathrm{KDP}(101)$ & 8 & 0.51 & 20 & $4.2 \times 10^{-14}$ & 1.1 & 650 \\
Thaumatin & 1,552 & 5.5 & 0.4 & $1.3 \times 10^{-13}$ & 1.2 & 800 \\
Canavalin & 10,660 & 7.4 & 2 & $1.2 \times 10^{-12}$ & 12.2 & 800 \\
Catalase & 16,436 & 11.7 & 0.32 & $5.2 \times 10^{-13}$ & 2.5 & $<2000$ \\
\hline
\end{tabular}

where $N_{c}$ refers to the number of molecules in a critical nucleus and $a$ is a unit cell dimension

nuclei, (2) the lateral spreading rate is constant and independent of direction, and (3) nuclei can be formed anywhere (i.e., on top of complete or incomplete layers). Ohara and Reid ${ }^{74}$ determined the resulting growth rate for this model to be

$$
G=h v^{2 / 3} I^{1 / 3}
$$

where $v$ is the rate of lateral spreading (methods for determining $v$ are given in §5.1.3). Cuppen et al. ${ }^{76}$ provide a detailed analysis of the birth and spread model for the case of directionally dependent rates of lateral spreading.

In order to obtain realistic growth rates for faces growing by this mechanism $(G \gtrsim 0.1 \mathrm{~nm} / \mathrm{s})$, Equation 20 limits the range of $\Delta G_{c}$ to small orders of magnitude (which implies large $\Delta \mu$ or small $\gamma_{\text {edge }} / R T$ ) as larger values of $\Delta G_{c}$ result in insignificant nucleation rates. Land and De Yoreo $^{66}$ report the lowest values of $\sigma$ at which growth by 2D nucleation was observed experimentally for various compounds, which are reproduced (in terms of $\alpha \equiv \frac{C}{C_{e q}}$ ) in Table 2. The apparent correlation between normalized edge energy and the onset of $2 \mathrm{D}$ nucleation and growth as the dominant growth mechanism is shown in Figure 9. When small organic molecules are crystallized by cooling, the upper limit on supersaturation is normally $\frac{C}{C_{e q}} \lesssim 1.65$ and the lower limit on $\gamma_{e d g e} a h \gtrsim 0.01 \times 10^{-12} \mathrm{erg}$, shown in the grey region in Figure 9. It is expected, therefore, that most $\mathrm{F}$ faces on organic crystals grown in a cooled environment will grow by the spiral mechanism. 


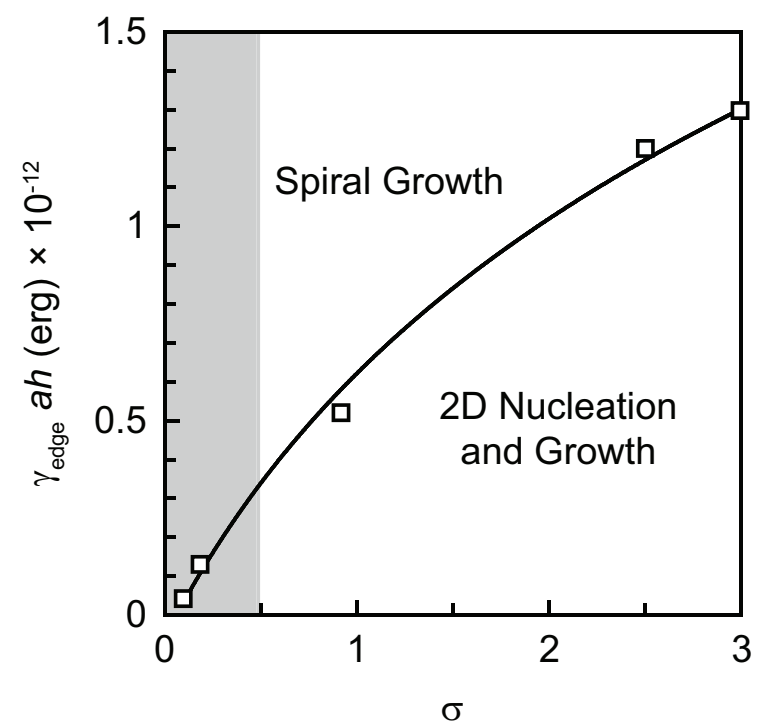

Figure 9: Relationship between the onset of 2D nucleation and normalized edge energy adapted from Land and De Yoreo ${ }^{66}$ (see Table 2). The grey region highlights an estimated region for crystallization of molecular organics from solution.

\subsubsection{Modeling Spiral Growth}

In 1931, Volmer and Schultze ${ }^{78}$ observed the growth of iodine crystals at $\alpha \sim 1.01$; which is far below the minimum supersaturation predicted for growth to occur by the $2 \mathrm{D}$ nucleation and growth mechanism. This study demonstrated the need for an alternative mechanism to explain layered growth at low supersaturations. This alternative came in the form of the spiral growth mechanism proposed by Frank which was based on the creation of renewable sources of steps at the emergence of screw dislocations on crystal faces. ${ }^{79}$ Working with Burton and Cabrera, this mechanism was further developed and published in their landmark paper. ${ }^{69}$ The model developed in that paper to describe the growth of crystal faces by a spiral mechanism is referred to as the BCF model.

This model results in a relationship between the perpendicular growth rate of the $h k l$ face, the step velocity and geometry of the face, given by

$$
G_{h k l}=\left(\frac{v_{i} h}{y_{i}}\right)_{h k l}
$$

where $v_{i}$ and $y_{i}$ are the step velocity and interstep distance for the $i^{t h}$ edge, respectively. The step height, $h_{h k l}$, is typically equivalent to the interplanar spacing in the direction normal to the face or a fractional $(<1)$ multiple thereof, depending on the growth unit. Equation 22 can also be 
re-written in terms of the characteristic rotation time, $\tau$, as

$$
G_{h k l}=\left(\frac{h}{\tau}\right)_{h k l}
$$

where $\tau$ is the time it takes for the first turn of the spiral to complete a single revolution. Therefore, each time the spiral completes a revolution the perpendicular distance of the face from the center of the crystal is increased by an amount $h_{h k l}$. Methods for predicting relative $\tau$ for $\mathrm{F}$ faces, based on spiral geometries and step velocities are developed by Snyder and Doherty. ${ }^{80}$

\subsubsection{Step Velocities}

Step velocities can be determined from a detailed mass balance at the step front (see De Yoreo and Vekilov ${ }^{77}$ or $§ 3.2 .1$ of Markov ${ }^{81}$ for details) when growth is limited by surface integration. Assuming the concentration of solute at the surface is equivalent to that of the bulk (i.e., $C_{s}=C_{\infty}$, $\delta_{d} \rightarrow 0$ ), the resulting velocity for the $i^{\text {th }}$ step in the surface integration limit is given by

$$
v_{i}=V_{M}\left(C_{\infty}-C_{e q}\right) a_{p, i} \rho_{k, i} \bar{\nu} \exp \left(-\frac{\Delta U}{R T}\right)
$$

where $a_{p, i}$ is the distance the $i^{\text {th }}$ step propagates by the addition of a row of molecules, $\bar{\nu}$ is a frequency factor, $\Delta U$ is the energy barrier for incorporation (which can be approximated by the desolvation enthalpy) and $\rho_{k, i}$ is the kink density along the $i^{\text {th }}$ step given by $\rho_{k, i}=a_{e, i} / x_{0, i}$ where $a_{e, i}$ is the distance along the step that a single growth unit occupies and $x_{0, i}$ is given by Equation 14. Equation 24 is typically expressed in terms of the step kinetic coefficient, $\beta_{i}$, defined by $\beta_{i} \equiv a_{p, i} \rho_{k, i} \bar{\nu} \exp \left(\frac{-\Delta U}{R T}\right)$, resulting in

$$
v_{i}=V_{M}\left(C_{\infty}-C_{e q}\right) \beta_{i}
$$

Combining Equations 25 and 22 results in a growth rate by spirals for the $h k l$ face in the surface integration limit (denoted by the subscript $S I$ ) given by

$$
G_{S I, h k l}=V_{M}\left(C_{\infty}-C_{e q}\right)\left(h \beta_{i} / y_{i}\right)_{h k l}
$$

Doherty and co-workers ${ }^{75,82}$ have applied the surface integration limit to predict the relative face growth rates for solution grown molecular organic crystals. Since only relative growth rates are required for shape predictions, the isotropic terms in Equation 24 (terms without subscript $i$ ), 
Table 3: Typical values for molecular organic crystals, used to estimate $\beta$ and $v$.

\begin{tabular}{cccccc}
\hline$V_{M}\left(C_{\infty}-C_{e q}\right)$ & $\rho_{k}$ & $a_{p}$ & $h$ & $\bar{\nu}$ & $\Delta U$ \\
& & $(\AA)$ & $(\AA)$ & $(\mathrm{Hz})$ & $(\mathrm{kcal} / \mathrm{mol})$ \\
\hline 0.005 & 0.05 & 5 & 5 & $10^{13}$ & 7.5 \\
\hline
\end{tabular}

do not need to be determined. ${ }^{82}$ Under these conditions, the predicted relative velocity of a step in the $i^{\text {th }}$ direction is given by:

$$
v_{\infty, i} \sim a_{p, i}\left(\frac{a_{e}}{x_{0}}\right)_{i} \sim \frac{a_{p, i}}{1+1 / 2 \exp \left(\phi_{k i n k, i} / R T\right)}
$$

Kink energy is therefore a key anisotropic physical property in a morphology prediction. Thus, methods have been developed for calculating kink energy, including contributions from both solid and solution phases. ${ }^{75,82-84}$

Typical values for molecular organic crystals of the parameters in Equations 24-27 are listed in Table 3. These values can be used to provide order of magnitude estimates for $\beta$ and $v$ resulting in $\beta=\mathcal{O}(1,000 \mu \mathrm{m} / \mathrm{s}), v=\mathcal{O}(5 \mu \mathrm{m} / \mathrm{s})$ when surface integration is rate limiting. Assuming $h=5 \AA$ and $y=\mathcal{O}(100 \mathrm{~nm})$ results in $G_{S I}=\mathcal{O}(25 \mathrm{~nm} / \mathrm{s})$, which is in agrement with the values determined for growth of $\mathrm{F}$ faces on a paracetamol crystal from aqueous solutions. ${ }^{85,86}$

The Damköhler number, $D a$, which can be used to determine whether growth on a face is limited by surface integration or bulk transport, is obtained by forming the ratio of the growth rates from Equations 26 and 13

$$
D a \equiv \frac{G_{S I, h k l}}{G_{B T, h k l}}=\frac{\left(h \beta_{i} / y_{i}\right)_{h k l}}{\mathcal{D} / \delta_{d, h k l}}
$$

For $D a \ll 1, G_{B T} \gg G_{S I, h k l}$ and the growth is surface integration limited (with the rate given by Equation 26); conversely for $D a \gg 1, G_{B T} \ll G_{S I, h k l}$ and the growth rate is bulk transport limited (with the rate given by Equation 13).

Using the estimate for $\beta$, obtained for small molecule molecular organic crystals, and assuming $y=\mathcal{O}(100 \mathrm{~nm}), \mathcal{D}=10^{-5} \mathrm{~cm}^{2} / \mathrm{s}$ and $\delta_{d}=10 \mu \mathrm{m}$ yields $D a=\mathcal{O}(0.05)$. Thus indicating that in the supersaturation regime where spiral growth is the dominant mechanism for most molecular organics grown in an industrial crystallizer (or in any crystallizer where $\delta_{d} \leq \mathcal{O}(10 \mu \mathrm{m})$ ), growth will be limited by the kinetics of surface integration processes. Vekilov and Alexander ${ }^{87}$ performed a similar analysis for protein crystals grown in a natural convection environment (where 
it was assumed that $\delta_{d}=300 \mu \mathrm{m}$ ) and obtained $D a$ ranging from 0.05 for lysozyme to 1.2 for satellite tobacco mosaic virus.

\subsubsection{Step Velocity Profiles and Interstep Distances}

The step velocity profile (relationship between edge length, $l$, and step velocity) for a polygonized spiral has typically been modeled by one of two limiting cases; the thermodynamic case (using the Gibbs-Thomson relationship) or the profile proposed by Voronkov. ${ }^{88}$ The Gibbs-Thomson relationship predicts ${ }^{89}$ :

$$
v(l)=\left\{\begin{array}{cl}
0 & l \leq l_{c} \\
v_{\infty}\left(1-\frac{e^{\sigma\left(l_{c} / l\right)}-1}{e^{\sigma}-1}\right) & l>l_{c}
\end{array}\right\}
$$

which for low supersaturation reduces to the commonly used form:

$$
v(l)=\left\{\begin{array}{cc}
0 & l \leq l_{c} \\
v_{\infty}\left(1-l_{c} / l\right) & l>l_{c}
\end{array}\right\}
$$

Though the Voronkov ${ }^{88}$ profile was developed for low supersaturations, it has been experimentally shown to apply at larger values of $\sigma$ than one might expect. This profile is given by;

$$
v(l)=\left\{\begin{array}{cc}
0 & l \leq l_{c} \\
v_{\infty} & l>l_{c}
\end{array}\right\}
$$

The velocity profiles (Equations 29 and 31) approach each other as supersaturation increases. The effects of different velocity profiles on growth rates were first discussed by Burton et al.. ${ }^{69}$ Recently Chernov ${ }^{90,91}$ has examined the validity of Equations 30 and 31 in light of in situ AFM observations of growing steps on various materials. ${ }^{89,92,93}$ Observations of step velocities for calcite performed by Teng et al. ${ }^{89}$ are reproduced here in Figure 10a. As shown in the figure, the velocity profile is independent of supersaturation, which is in disagreement with velocity profile predicted using the Gibbs-Thomson relationship (Equation 29).

Interstep distances can be determined from the geometries (including angles and critical lengths) and velocities (including velocity profiles) of a spiral's steps. ${ }^{80}$ In doing so, Burton et al. ${ }^{69}$ assumed a round Archimedean spiral comprised of steps with velocity profiles consistent with Equation 30. Applying these assumptions they found $y=4 \pi r_{c}$ for their calculation of interstep distance, where $r_{c}$ is the critical radius of a disk-shaped 2D nucleus. Cabrera and Levine ${ }^{94}$ determined $y=19 r_{c}$ 
(a)

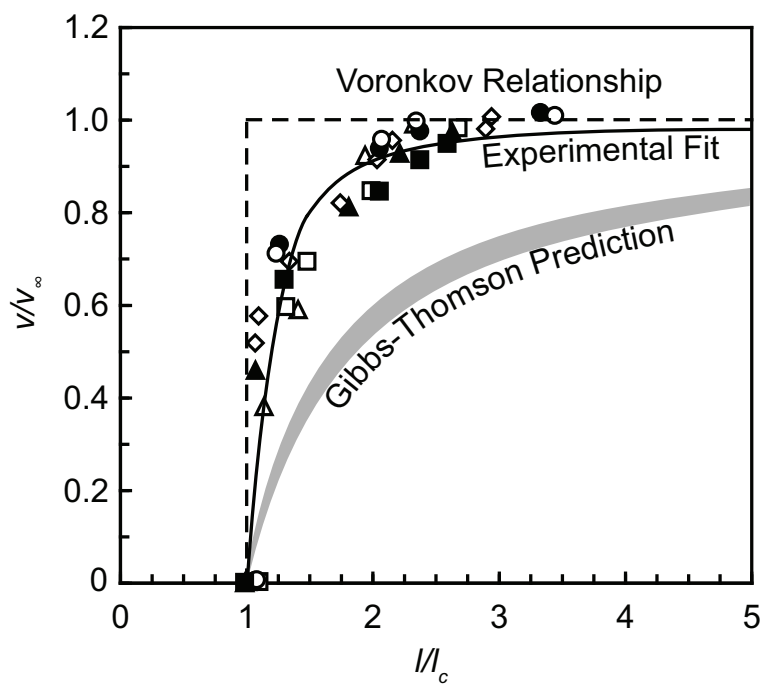

(b)

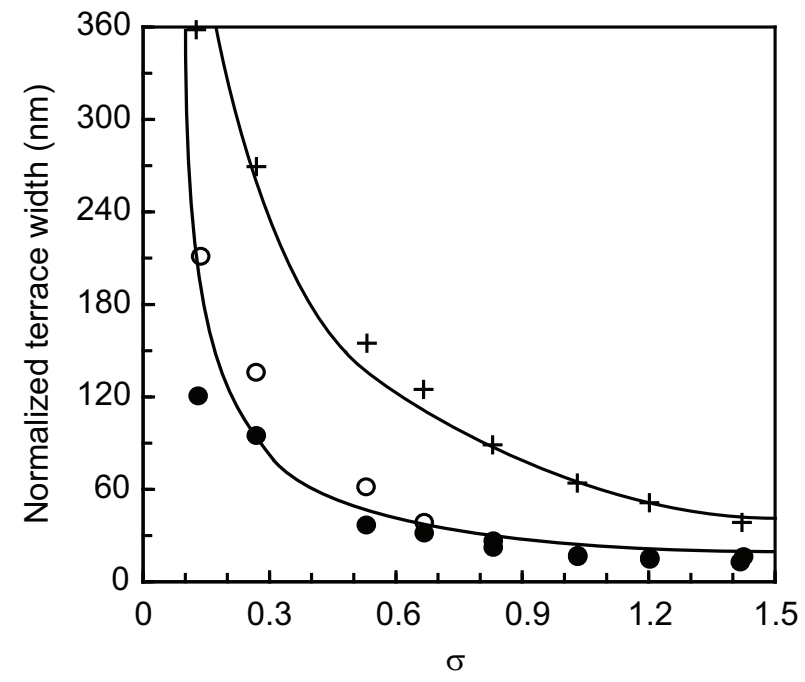

Figure 10: The velocity profile at different supersaturations (a) and the relation between supersaturation and $y$ (b) for calcite, adapted from Teng et al.. ${ }^{89}$ In (a) the symbols are experimental data taken with $\sigma$ in the range from 0.346 to 0.826 . In (b) the circles are experimental data and the crosses are the $y \simeq 9.5 l_{c}$ prediction.

for similar spirals; this relationship was used by Liu et al. ${ }^{83}$ for crystal shape predictions and is commonly cited throughout the literature. Since $r_{c} \sim \sigma^{-1}, y \sim \sigma^{-1}$ using either the Burton et al. ${ }^{69}$ or the Cabrera and Levine ${ }^{94}$ prediction.

Teng et al. ${ }^{89}$ and Chernov ${ }^{91}$ discuss the effect of the velocity profile on isotropic square spirals. For square spirals with step velocity profiles conforming to Voronkov's profile (Equation 31 ), $y=4 l_{c}$ (see Figure 7), whereas square spirals with step velocity profiles that conform to Equation 30, result in $y \simeq 9.5 l_{c}$. In situ AFM observations of calcite growth ${ }^{89}$ have demonstrated steps advancing with $y \sim \sigma^{-1}$ (Figure $10 \mathrm{~b}$ ) and $y \approx 4 l_{c}$ indicating the usefulness of Voronkov's ${ }^{88}$ relationship. Assuming square spirals that obey Equation 31, interstep distances will range from $y=\mathcal{O}(100-1,000) \mathrm{nm}$ for $\gamma_{\text {edge }}=25-100 \mathrm{erg} / \mathrm{cm}^{2}, V_{M}=150 \mathrm{~cm}^{3} / \mathrm{mol}$ and $\sigma=(0.01-0.1)$.

\subsection{Crystal Simulations}

Simulation techniques are also valuable tools for use in crystal growth modeling. Some of the earliest simulation results for crystal growth were those of Gilmer and Bennema, where the dynamics and 
transition probabilities of surface diffusion in crystal growth were determined for the (100) face of a Kossel crystal. ${ }^{95}$ Since that time, simulation techniques including Monte Carlo, kinetic Monte Carlo and molecular dynamics simulations have been applied to crystal growth for a variety of molecules. See the reviews of Rohl ${ }^{96}$ and van der Eerden ${ }^{97}$ for the development of simulation techniques. More recently, several groups have continued to advance the field of crystal growth via simulations. The Meekes group has continued to evolve their Monte Carlo algorithm to include more complex and generic systems such as fats. ${ }^{98}$ Gilmer continues to develop crystal growth simulations using a lattice Monte Carlo approach, most recently for a high explosive pentaerythritol tetranitrate (PETN). ${ }^{99}$ Additionally, Piana et al. have developed a kinetic Monte Carlo scheme and have applied it to the growth of urea crystals from water. ${ }^{100}$ A similar scheme was followed by Anderson et al. ${ }^{101}$ to predict the shape of hydrothermally synthesized zeolite A crystals. These simulation techniques have both advantages and drawbacks compared to the modeling methods described in $\S 5.1$ for problems ranging from fundamental discovery to product and process development.

Molecular dynamics simulations can be extremely useful in determining the fundamental mechanisms and movements of molecules as they incorporate into the crystal. The difficulty in applying these molecular dynamics schemes to crystal growth is the disparity in the relevant time and length scales between processes. For example, considering a molecular crystal surface of $\mathcal{O}(1000)$ molecules $\mathcal{O}(10$ by 10$) \mathrm{nm}$, a molecular dynamics simulation of $1 \mathrm{~ns}$ would take about $\mathcal{O}(10)$ hours of single processor computation time. Kinetic Monte Carlo (KMC) schemes can allow for calculations on more realistic time and length scales (micron size crystals in $\mathcal{O}(10)$ hours of computation time); however, in order to implement these schemes the Monte Carlo moves need to be defined. These moves are often determined using underlying molecular dynamics schemes ${ }^{99,100}$ or other move determining methods which require previous knowledge or good intuition about the specific system. Thus, in order for calculations to be performed over a range of conditions (i.e., multiple solvents), the MD and KMC codes must be rerun. It is this set of challenges of time scales that limits the ability to interlink simulation methods into process and product design methodologies. Simulation techniques, however, are excellent tools for discovering the underlying physics and solid state chemistry of the processes that occur during crystal growth. The insight gained can be used to improve the fidelity of mechanistic models described in $\S 5.1$. 


\section{$5.3 \quad$ Experiments}

A variety of microscopy techniques have been used to investigate crystallization phenomena. A summary of these techniques adapted from ${ }^{102}$ is given in Table 4.

Optical microscopy is a commonly used technique to characterize the morphology of crystals $\gtrsim 1 \mu \mathrm{m}$ both in situ and ex situ. It is often the first microscopy technique used to characterize a crystallization product. A number of temperature controlled flow cell investigations have been performed in situ to determine the growth shapes of various small molecule molecular organic crystals. ${ }^{103-105}$ The kinetics of polymorphic phase transformations in drug solutions have been studied in situ using a quiescent thermostated batch crystallizer placed under an optical microscope by Veesler et al.. ${ }^{106}$ While the spatial resolution of optical microscopy is limited by the wavelength of light, it offers the ability to image growth processes in situ at the length scale equivalent to that of crystal faces.

Interferometry provides the ability to measure the steepness of the surface and face growth (or dissolution) rates simultaneously. Interferometry provides a higher vertical resolution $(\sim 1 \mathrm{~nm})^{107}$ than optical microscopy, allows for faster growth rates and can be used to study larger areas than in situ AFM. ${ }^{108}$ However, the technique is limited by relatively poor $(\sim 1 \mu \mathrm{m})$ lateral resolution making it unable to resolve individual steps. ${ }^{108,109}$ Interferometric studies have been performed on a number of macromolecular crystals, ${ }^{87,110} \mathrm{KH}_{2} \mathrm{PO}_{4}$ (ADP) ${ }^{111,112}$ and small molecule organic crystals such as paracetamol, ${ }^{85}$ amongst others. Unique to interferometry is the ability to observe solute concentration iso-contours near crystal surfaces. These observations allow for the determination of depletion boundary layer thicknesses. ${ }^{112}$

Investigations into solution crystal growth are limited to ex situ observations when using any of the various electron microscopy techniques as they are all operated under vacuum. Electron microscopy (specifically scanning electron microscopy) has traditionally been used to determine crystal shapes. However, modern field emission scanning electron microscopes (FE-SEM) can use low accelerating voltages $(\sim 0.5-1.5 \mathrm{kV})$ that enable the study of surface topography on non-conducting materials. Low accelerating voltage FE-SEM studies have provided clear images of growth terraces and spirals on faces of zeolite and zeo-type materials. ${ }^{113-115}$ These studies have been important in determining growth mechanisms for these systems. ${ }^{114-116}$

Atomic force microscopy has been used to characterize the surface microstructure of a variety of 
Table 4: Microscopy techniques used to determine crystallization kinetics and growth mechanisms.

\begin{tabular}{|c|c|}
\hline Technique & Information Obtained \\
\hline Optical Microscopy & $\begin{array}{l}\text { - applied for in situ or ex situ characterizations of morphology } \\
\text { - characterize additive/solvent interactions with specific faces } \\
\text { - identify growth mechanisms (layered or rough) } \\
\text { - measure crystal face growth rates } \\
\text { - monitor shape evolution and polymorph transformations }\end{array}$ \\
\hline Interferometry & $\begin{array}{l}\text { - applied for in situ examination of morphology and surface } \\
\text { steepness } \\
\text { - simultaneously measure step velocities and face growth rates } \\
\text { - identify growth mechanisms (layered or rough) } \\
\text { - determine solute concentration iso-contours and depletion } \\
\text { boundary layer thicknesses }\end{array}$ \\
\hline Electron Microscopy & $\begin{array}{l}\text { - applied for ex situ examination of morphology and surface } \\
\text { topography } \\
\text { - characterize shape and additive/solvent interactions with specific } \\
\text { faces } \\
\text { - identify growth mechanisms (spirals, 2D nuclei or rough) }\end{array}$ \\
\hline Atomic Force Microscopy & $\begin{array}{l}\text { - applied for in situ or ex situ characterizations of surface } \\
\text { topography } \\
\text { - characterize additive/solvent interactions with specific faces } \\
\text { - identify growth mechanisms (spirals, 2D nuclei or rough) } \\
\text { - determine step kinetic coefficients } \\
\text { - determine kink densities (macromolecular systems) }\end{array}$ \\
\hline
\end{tabular}


different crystalline systems including: ionic crystals, ${ }^{89,108,117-123}$ porous materials, ${ }^{101,114,116,124-127}$ macromolecules and proteins ${ }^{128-130}$ and small molecule organics. ${ }^{131-134}$ Atomic force microscopy provides the ability to image crystal growth and dissolution processes in situ at nanometer-scale resolutions. This ability has been used to investigate several fundamental aspects of crystal growth including: methods of kink formation, ${ }^{135-138}$ step velocity profiles, ${ }^{89}$ the transition between growth mechanisms ${ }^{66}$ and the effects of additives and impurities. ${ }^{121-123,131,132,139}$ AFM has also been used to distinguish between polymorphs for pharmaceutical compounds ${ }^{140}$ and to observe nucleation in proteins. ${ }^{5}$

\section{Solvents, Impurities and Additives}

The importance of solvent selection in a crystallization process can be traced to the fact that the nature of the solvent-solute interactions at the crystal surface greatly influence the ultimate shape of the crystal. Wells drew attention to these phenomena by citing solvent effects on the crystal shapes of iodoform and anthranilic acid ${ }^{141}$ and resorcinol ${ }^{141,142}$ (see also Davey et al. ${ }^{143}$ for more on resorcinol). The crux of the matter is that a given crystallographic orientation on a crystal exposes a particular set of atoms of the solute molecule, so different orientations therefore have varying affinities for the solvent; these varying affinities impart different growth rates to the crystal faces and thus influence the shape. Other examples of such behavior are abundant in the literature. For instance, Davey et al. ${ }^{144}$ demonstrated that succinic acid grown from water resembles a flat, diamond-like plate and exhibits large $\{001\}$ basal planes bounded by $\{110\}$ side faces, while succinic acid grown from isopropanol resembles an elongated hexagon with $\{001\}$ basal planes and bounded by the $\{110\}$ and $\{010\}$ side faces. Gordon and $\operatorname{Amin}^{145}$ disclosed that ibuprofen grown out of more polar solvents such as methanol or isopropanol yield crystals with a more equant aspect ratio, whereas ibuprofen crystals grown out of hexane yield much more elongated crystals. Finally, Davey ${ }^{146}$ and Lahav and Leiserowitz ${ }^{147}$ have reviewed several other examples of solvent-influenced crystal shapes and discuss mechanisms related to the solvent effect.

It is also well known that foreign molecules (additives or impurities) influence the growth shape of crystals. These molecules can be divided into three categories: tailor made additives, small molecule additives and macromolecules. Small molecule additives include surfactants and fatty acids. Assuming the crystallizing solute has a molecular weight no more than 200-500 atomic mass 
units, a macromolecular additive or impurity could be a protein, polymer or peptide. An important type of molecular impurity or additive is one which is structurally related to the solute, sometimes referred to as being "tailor-made." As the name suggests, a tailor-made additive is a molecule that is practically identical to the crystallizing solute molecule; these types of additives are relevant to crystallization as they often occur as reaction by-products formed during the synthesis of the solute. There are many reports in the literature that describe the effect of additives or impurities on the shape or growth of crystals, both organic and inorganic. ${ }^{123,148-151}$ Sections 6.1 - 6.3 describe some of these experiments, as well as past modeling efforts.

\section{1 "Tailor-Made" Additives}

Weissbuch et al. ${ }^{152}$ demonstrated that chiral molecules can be directly assigned an absolute configuration (levorotary "L" or dextrorotary "D") based on changes they caused to the morphology of a crystal composed of a prochiral molecule (i.e., an achiral molecule that can be converted to a chiral molecule in a single step) as the crystal is grown in the presence of either resolved or racemic molecular additives. In particular, the $\alpha$ polymorph of glycine $\left(\mathrm{H}_{3}^{+} \mathrm{NCH}_{2} \mathrm{COO}^{-}\right)$, when crystallized out of water, normally exhibits a coffin shape whose basal planes are the $\{010\}$ family (i.e., the (010) and (010) planes); the glycine molecules on these planes are related by a center of inversion. Ultimately, these researchers observed that if D-alanine $\left(\mathrm{CH}_{3} \mathrm{CH}\left(\mathrm{NH}_{2}\right) \mathrm{COOH}\right)$ was present in solution during crystallization, the $\alpha$-glycine crystal possessed a much larger (010) plane as compared to the $(0 \overline{1} 0)$ face; in contrast, the crystal possessed a large $(0 \overline{1} 0)$ face if L-alanine were in solution. Their results are shown in Figure 11a-c. The effects of the D- and L-alanine on crystal shape were so pronounced that the researchers were able to assign their absolute chirality.

A U.S. Patent by Klug and van Mil ${ }^{154}$ described the effect of properly chosen additives on the purity of adipic acid as it crystallized from water. They employed both surfactants and tailor-made additives and observed that certain additives caused a change in the normal shape of adipic acid such that crystals with the altered shape exhibited improved purity with respect to the uptake of other structurally related molecules. In particular, the authors observed that when adipic acid, whose steady state convex hull is bounded by the (dominant) $\{100\},\{001\}$, and $\{011\}$ forms, was crystallized from water in the presence of an amount on the order of $1 \mathrm{wt} . \%$ (solvent-free basis) of any of glutaric acid $\left(\mathrm{HOOC}\left(\mathrm{CH}_{2}\right)_{3} \mathrm{COOH}\right)$, succinic acid $\left(\mathrm{HOOC}\left(\mathrm{CH}_{2}\right)_{2} \mathrm{COOH}\right)$, maleic acid 


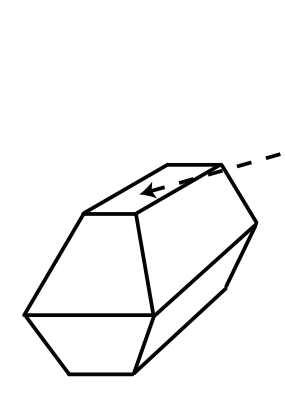

(a)

\section{$\underline{\alpha-g l y c i n e}$}

(010)

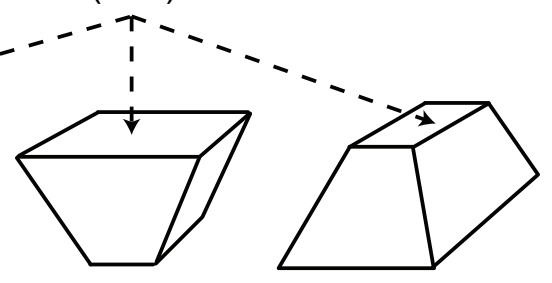

(b)

(c)

D-alanine L-alanine

$\underline{\text { adipic acid }}$

(100)

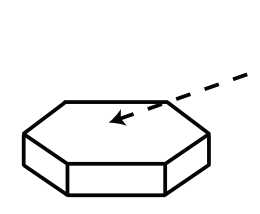

(d)

native

(e)

TMDAC

(f)

SDBS

Figure 11: Growth shapes of crystals of $\alpha$-glycine (a-c) ${ }^{152}$ and adipic acid (d-f) ${ }^{153}$ as influenced by additives. 
(cis-HOOC $(\mathrm{CH})_{2} \mathrm{COOH}$ ) or fumaric acid (trans-HOOC $\left.(\mathrm{CH})_{2} \mathrm{COOH}\right)$. The resultant crystals were hardly distinguishable from the native crystal; that is, the $\{100\}$ form still comprised most of the total area. However, when 3 wt.\% (solvent-free basis) of caproic acid $\left(\mathrm{CH}_{3}\left(\mathrm{CH}_{2}\right)_{4} \mathrm{COOH}\right)$ was present, the adipic acid crystals exhibited a shape where the $\{001\}$ form dominated. Klug and van Mil proposed that caproic acid, and no other additive, was incorporated into the $\{001\}$ face and interfered with the subsequent addition of adipic acid molecules; moreover, they also observed a simultaneous reduction in the uptake of the other molecules.

These experiments demonstrate that both tailor-made additives or impurities can have a significant impact on shape, which arises from the specificity of interaction between the crystal's surfaces and the additive or impurity. The specificity ultimately arises out of the high degree of similarity between the solute molecule and the tailor-made additive or impurity. Additionally, shape is seen to be important to the most basic purpose of crystallization: separation of a particular species. Therefore purity of the crystallized solute is important, and a well-chosen additive can alter the shape so as to prohibit uptake of structurally-related impurities.

\subsection{Macromolecules}

Michaels and Colville ${ }^{155}$ presented results from a systematic study on the morphology change exerted by two surfactants, anionic sodium dodecylbenzene sulfonate (SDBS) and cationic trimethyldodecylammonium chloride (TMDAC), on adipic acid as it was crystallized from water. The authors observed that when adipic acid was grown in the presence of 50 ppm SDBS, the $\{001\}$ and $\{011\}$ forms became increasingly dominant over the $\{100\}$ form. At higher concentrations of SDBS (500 ppm), the $\{100\}$ form had almost disappeared and the crystals resembled thin rods or needles with $\{001\}$ and $\{011\}$ side faces. On the other hand, when adipic acid was grown with 96 ppm TMDAC the $\{100\}$ form became larger at the expense of the other forms. The authors determined that both SDBS and TMDAC reduced the growth rate of all forms, but the surfactants' effects on the different forms were anisotropic. Indeed, the measured growth rates revealed that TMDAC was much more effective in reducing the growth rate of the $\{100\}$ form as compared to either $\{011\}$ or $\{001\}$. Michaels and Tausch ${ }^{153}$ performed similar experiments, the results of which are reproduced here in Figure 11(d-f).

Another example of the effect of macromolecules on crystal growth has been observed in na- 
ture: Antarctic fish are able to survive in waters where temperatures fall as much as $1^{\circ} \mathrm{C}$ below the equilibrium freezing point of their body's fluids. Ice crystals are abundant in the waters at these temperatures. DeVries ${ }^{156}$ and Raymond and DeVries ${ }^{157}$ demonstrate that these fish have glycoproteins and serum proteins which prevent the growth of ice crystals in their blood. Unlike $\mathrm{NaCl}$, these "antifreeze" proteins do not lower the freezing point through a colligative effect, as evidenced by the fact that the melting point of ice crystals in the fishes' blood serum is virtually unchanged. Rather, the proteins preferentially adsorb to nascent ice crystals and disrupt growth.

\subsection{Modeling Impurity and Additive Effects}

Cabrera and Vermilyea ${ }^{158}$ advanced the idea that flowing steps could be stopped by additives which adsorb to a crystal surface and remain immobile (the term immobile is relative; more specifically, the additive must have an adsorption lifetime much longer than some characteristic time for growth). Their hypothesis was that flowing steps would be forced to percolate through a collection of additives; as the edge encountered the immobile additive, the edge would be "pinned" at the point of contact and a segment of edge between two additives would begin to curve as growth proceeded. If the spacing between the additives parallel to the edge direction was less than some critical length $l_{c}$ (see §5.1.1), the steps would be partitioned into sections of less-than-critical length and growth would be arrested. If the additives were spaced a distance greater than $l_{c}$, the step would pass between the additives and continue to grow; however, due to its curvature the edge would experience a decrease in the driving force for growth resulting in a diminished velocity. As shown in $\S 5.1 .1$ the critical radius of a 2-dimensional nucleus $r_{c} \sim \sigma^{-1}$. Therefore, one consequence of Cabrera and Vermilyea's model is that there will be some critical supersaturation $\sigma^{*}>0$ below which growth cannot occur (sometimes called a "dead zone"). Physically this means that by decreasing the supersaturation to some non-zero value, $\sigma^{*}$, the critical length becomes larger than the spacing between the additives, and growth is arrested. Several accounts in the literature report observation ${ }^{132,159}$ of this effect.

Some additives may have adsorption lifetimes comparable to or shorter than the characteristic time for surface growth; in these cases, the above model does not hold. Because the velocity of a step is directly proportional to its kink density (Equation 27), a natural alternative is to consider the possibility that additives adsorb into kink sites and reduce their effective density. An 
equivalent statement is to consider that the additives increase the average distance between effective kink sites along an edge. Chernov ${ }^{160}$ and Cabrera and Coleman ${ }^{161}$ developed such an expression for a crystal growing from a vapor phase with additive partial pressure, $P_{p}$, by considering the relative equilibrium probabilities of finding each of the following along an edge: a straight segment, a kinked segment, a straight segment with an adsorbed additive, and an additive adsorbed at a kink site. If step velocity is proportional to kink density, (see §5.1.3) as given by Equation 27, then the (poisoned) kink density must relax to its equilibrium value on a timescale much shorter than some characteristic timescale for growth of the edge. Physically this leads to the requirement that 1-dimensional particle exchange along the edge is much faster than 2 or 3 -dimensional mass transport between the edge and the adlayer and/or the bulk phase.

Clydesdale et al. ${ }^{162,163}$ extended the attachment energy model (see $\S 3$ ) of crystal shape prediction $^{57,164}$ by considering the effects of tailor-made additives on the attachment energies. Their approach was to "map" the atomic coordinates of a tailor-made additive molecule onto those of a host or solute molecule and calculate slice and attachment energies, both when the imposter was within and external to a particular layer. Modified attachment energies were implemented in a shape model to yield shapes different from those predicted by the native model. The researchers employed a quantity developed by Berkovitch-Yellin ${ }^{165}$ called the "binding energy", $\Delta b$, which allowed them to determine which faces would favorably receive imposter molecules. The binding energy compares the energies of a host molecule after being bound up in a layer, $E_{\text {slice }}$, and subsequent attachment of the layer to a substrate, $E_{\text {att(-) }}$, to those of an imposter molecule bound up in the same layer, $E_{\text {slice }}^{\prime}$, and subsequent attachment of the layer to a substrate, $E_{\text {att(-) }}^{\prime}$. Defining $\Delta b \equiv E_{\text {slice }}^{\prime}+E_{\text {att(-) }}^{\prime}-\left(E_{\text {slice }}+E_{\text {att (-) }}\right)$, values of $\Delta b>0$ imply unfavorable imposter binding, values of $\Delta b<0$ imply favorable imposter binding, and values of $\Delta b=0$ imply indifferent imposter binding.

The step-pinning model of Cabrera and Vermilyea ${ }^{158}$ and the kink density reduction model of Chernov ${ }^{160}$ and Cabrera and Coleman ${ }^{161}$ address the problem of predicting the effect of additives or impurities on crystal growth by considering which mechanisms must be at work. In the case of the step-pinning model, one must know the extent to which additives or impurities have adsorbed on a crystal face. The kink density reduction model requires estimating the chemical potential of the additive or impurity in a kink site. The modified attachment energy model of Clydesdale 
et al., ${ }^{162,163}$ though helpful for characterizing crystal faces with respect to their ability to receive tailor-made additives or impurities in a heuristic fashion, does little to elucidate exactly how the growth rate of a face is reduced. A common approach to modeling the effects of additives or impurities is to assume a mechanism such as the ones described above, assume a form of an adsorption isotherm, perform experiments and then regress the data in order to estimate all the unknown parameters in the model equations. While this approach may correlate the measured data in a satisfactory manner, it is unlikely to result in a predictive methodology. A first-principles model is more desirable, which would predict everything, from the level of surface adsorption of the additives or impurities to the reduced growth rate and also account for any kind of growthreduction mechanism. Such a model can only spring from an understanding of the processes that govern the movements of molecules in and out of the surface during crystallization.

\section{Crystal Shape Evolution Model}

Whether growing from a pure solution or in the presence of additives or impurities, crystals can eventually reach a steady state shape; that is, the shape of the crystal has the property of being self-similar, even as it continues to grow. However, in a crystallizer, crystals do not all necessarily reach their steady state shape, nor does the shape of a crystal remain constant during growth. Consequently, it is important to have a method that predicts the evolving shape of a crystal grown from an arbitrary starting shape (e.g., seed) at any subsequent moment under realistic operating conditions. This method, under the appropriate limiting conditions, should also show that a crystal possesses a steady state shape.

\subsection{Geometric View of Crystal Growth}

A geometric view of surface evolution means that the outward normal velocity of a surface depends only upon its local orientation. The physical implication is that each point on the surface evolves in only one direction (forward), unlike, for instance, diffusive phenomena. The governing equation for geometric surface evolution is the Hamilton-Jacobi equation; the solution(s) to this equation describes many types of phenomena, ${ }^{166-168}$ including the growth of crystals. ${ }^{50}$ Solutions to the Hamilton-Jacobi equation exhibit so-called shock transitions which, for crystal growth, are 
interpreted as transitions from smooth to faceted growth. However, by assuming that crystals are faceted for all time, Zhang et al. ${ }^{52}$ developed a more straightforward and readily applicable evolution model as follows.

Let $G_{h k l}$ be the perpendicular growth rate of the $h k l$ face; it follows that the rate of change of the perpendicular distance $H_{h k l}$ from an origin inside the crystal to the face is given by:

$$
\frac{d H_{h k l}(t)}{d t}=G_{h k l}
$$

where this equation applies to each of $N$ faces. Zhang et al. ${ }^{52}$ nondimensionalize Equation 32 by selecting a reference face and using its corresponding values of $H_{r e f}$ and $G_{r e f}$ to define the relative perpendicular distance $x_{h k l} \equiv H_{h k l} / H_{r e f}$ and the relative growth rate $R_{h k l} \equiv G_{h k l} / G_{r e f}$. Coupled with a dimensionless time $d \xi=\left(G_{r e f} / H_{r e f}\right) d t$, they derive the following dynamical model for the remaining $N-1$ faces assuming the crystal is faceted for all time:

$$
\frac{d x_{h k l}(\xi)}{d \xi}=R_{h k l}-x_{h k l}
$$

where

$$
\xi=\ln \left(\frac{H_{r e f}(t)}{H_{r e f}(0)}\right)
$$

When all of the $R_{h k l}$ are constant, Equation 33 clearly displays a unique, stable steady state $\hat{x}_{h k l}$, given by

$$
\hat{x}_{h k l}=R_{h k l}
$$

Equation 35 is equivalent to the Frank-Chernov condition ${ }^{50,51}$ (see $\S 3$ ). A consequence of this steady state condition is that the steady state growth shape of a crystal can be predicted by knowing (either by measurement or prediction) the relative growth rates of each face. Conversely, the relative growth rates of each face can be obtained from the steady state growth shape. The growth models in $\S 5$ can be used to develop expressions for each $R_{h k l}$ and Equation 33 can be integrated to determine how the shape of a seed crystal (or a nucleus) evolves with time.

\subsection{Evolution Case Study: Ibuprofen}

Ibuprofen is a commonly used anti-inflammatory drug. When grown from aqueous solution, its crystal structure (as reported by Shankland et al. ${ }^{169}$ ) belongs to the monoclinic $P 2_{1} / c$ space group 
(a)

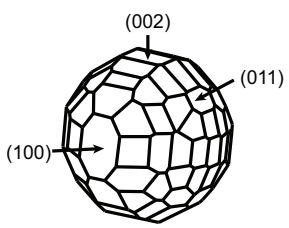

$(\xi=0$, seed $)$ (b)

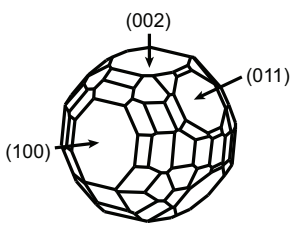

$(\xi=0.025)$ (c)

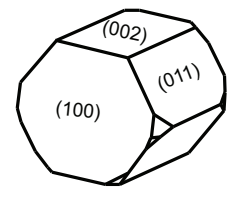

$(\xi=0.15)$ (d)

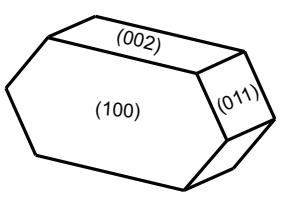

$(\xi \rightarrow \infty)$ (e)

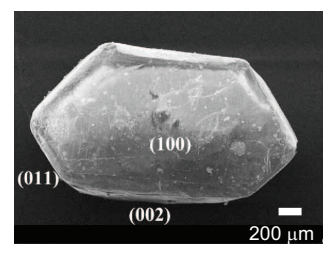

(experimental)

Figure 12: Predicted evolution of ibuprofen (a-d) $(\Delta \xi=0.025)$ and experimental steady state shape $^{170}(\mathrm{e})$, not drawn to scale.

with lattice parameters $a=14.397, b=7.818, c=10.506 \AA$ and $\beta=99.70^{\circ}$. Its unit cell is comprised of four molecules. Following the method of Winn and Doherty, ${ }^{75}$ the $\{100\},\{011\}$ and $\{002\}$ forms were determined to grow by the screw dislocation mechanism with relative perpendicular growth rates $R_{h k l}=1.00,2.67$, and 1.45, respectively. Other low index forms (e.g., $\{11 \overline{1}\}$ or $\{10 \overline{2}\})$ and higher index forms were assigned relative growth rates of 10 , as they contained no stable edges and are therefore expected to grow by the rough growth mechanism, which as noted in $\S 4.1$ is expected to occur at a rate at least 10 times faster than growth by a layered mechanism. The above relative growth rates account for the effect of the solvent.

To show the evolution of ibuprofen from an arbitrary state, a "spherical" seed was used as the initial condition for Equation 33. This seed was composed of 102 faces situated equidistantly from the origin (Figure 12a). The crystal was then evolved from this seed, assuming constant relative growth rates for all time $\xi$ and discretizing Equation 33 with a time step $\Delta \xi=0.025$. The results of this evolution are shown in Figure 12(a-d). As can be seen, the faster growing forms disappear, leaving only the $\{100\},\{011\}$ and $\{002\}$ forms on the steady state growth shape. The predicted steady state shape as $\xi \rightarrow \infty$ is shown in Figure 12d. It has a large hexagonal $\{100\}$ form that is slightly elongated in the $b$ direction because $R_{011}>R_{002}$. An experimental shape obtained by Cano et al. ${ }^{170}$ is shown in Figure 12e for comparison with the prediction.

It is important to recognize that the shape in Figure 12d is a pure prediction and nothing about the measured shape was used to obtain it. This example also demonstrates an important rule for crystal shape; faces with large perpendicular growth rates will usually possess smaller areas on the final shape. If faces have large enough growth rates, they will either disappear from the shape altogether or contribute to the formation of needle crystals. 


\section{Coupling Size and Shape Predictions}

So far we have described experiments, simulations and models for shape prediction and evolution of a single crystal growing from solution. However, when considering the design and control of industrial scale crystallizers the shape of the entire population of crystals becomes more relevant. There is often a particular interest in achieving a target particle size and shape distribution (PSD) of the crystal population, which is modeled by using population balance equations (PBE). ${ }^{171,172}$

\subsection{Multi-Dimensional Population Balances}

In order to track the distribution of crystal morphologies inside a crystallizer, shape effects need to be incorporated into PSD modeling. Iveson ${ }^{173}$ suggests that multi-dimensional population balances need to be used due to the limitations of conventional single dimensional PBE's that consider particle size in only one internal direction. For multi-dimensional population balances, a growth rate is needed for every independent direction that is used in the model; techniques for measuring growth rates in multiple directions are discussed in the following sections.

Several studies have developed two-dimensional PBEs in order track two characteristic crystal dimensions, usually length and width. Ma et al. ${ }^{174,175}$ used a compartmental model of a batch crystallizer to account for imperfect mixing, coupled with a 2-D PBE, to model the growth of needle-like potassium dihydrogen phosphate (KDP) crystals. For continuous crystallization, Puel et al. ${ }^{176-178}$ incorporated a detailed kinetic study of growth rates, primary and secondary nucleation and diffusion effects into a 2-D PBE model and were able to accurately predict the shape evolution of the rod-like hydroquinone crystals. Oullion et al. ${ }^{179,180}$ studied the batch cooling crystallization of a platelet-like organic crystals and included growth and secondary nucleation mechanisms into their 2-D PBE to determine the effects of seeding and cooling profiles on the final PSD. Other researchers have demonstrated the use of particle properties other than characteristic length in their multi-dimensional population balances. For instance, Gerstlauer et al. ${ }^{181}$ considered the internal lattice strain of individual crystals as a particle coordinate, and Briesen ${ }^{182}$ used a coordinate transformation on the multidimensional PBE to model the system by the crystal volume and a shape factor. Briesen demonstrated his particle coordinate transformation technique by simulating a batch cooling crystallizer and inverting the transformation to obtain the original two-dimensional number density function at the initial and final time as shown in Figure 13a. 
(a)

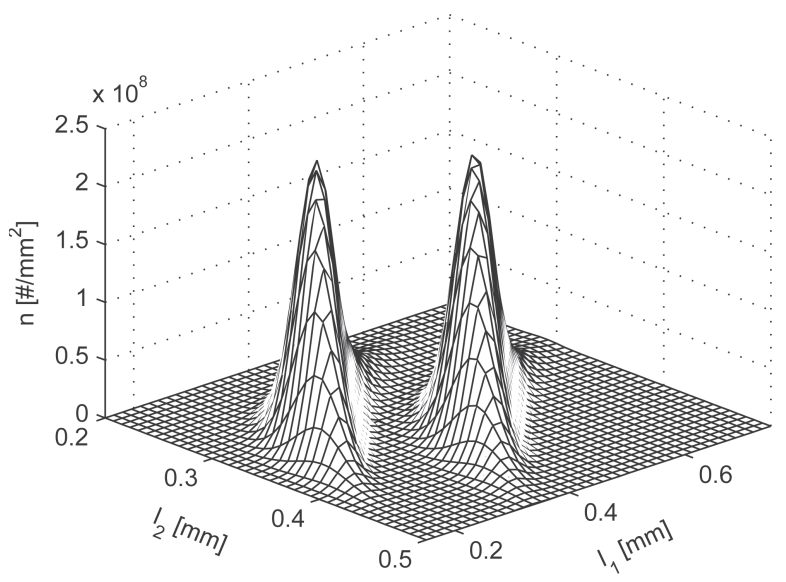

(b)

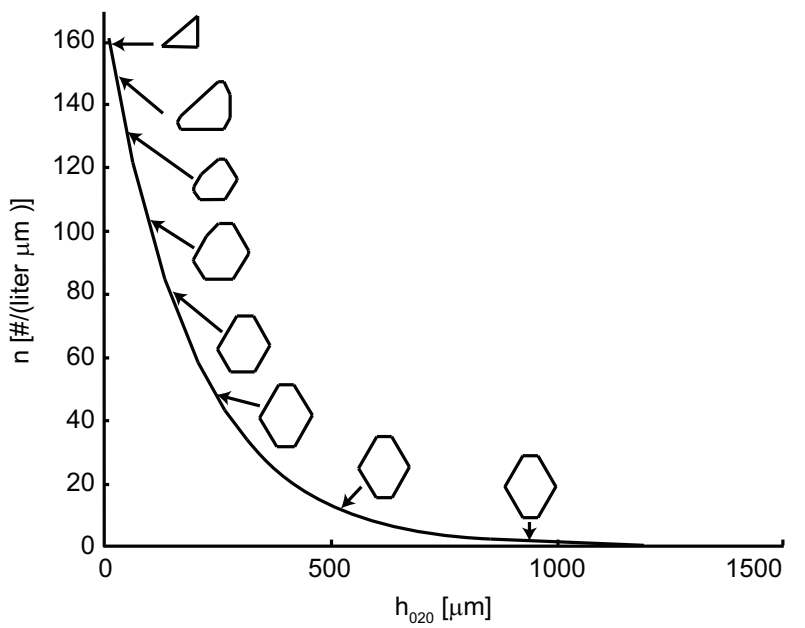

Figure 13: Multidimensional Population Balance Examples a) Two-dimensional representation with respect to the characteristic lengths of the particle number density function at the initial and final time $^{182}$ b) Steady-state crystal size and shape distribution for triangular seeds of succinic acid grown from water ${ }^{183}$

A different approached developed by Zhang and Doherty ${ }^{183}$ coupled a variation of the shape evolution model described in $\S 7$ with a 1-D population balance to simultaneously predict crystal shape evolution and size distribution. This method is applicable to crystals that can be treated as essentially 2D (e.g., plates) and assumes the relative growth rates of crystal faces are constant. This model requires only one absolute growth rate (for a reference face) and all others are found knowing the relative growth rates of all other faces relative to the reference face. This approach gives the size and shape evolution of every independent face. The method was applied to succinic acid grown from water as shown in Figure 13b.

Ma et al. ${ }^{184}$ have recently used polyhedral PBE modeling to predict the PSD for every independent crystal face. This model uses knowledge of the crystal shape of a single crystal as well as the measured growth rates for each independent family of planes; there are three sets of these planes in their study of potash alum. This prediction of individual facet growth at different times leads to the simultaneous prediction of crystal shape and size evolution.

One difficulty in implementing multi-dimensional PBEs is the increased computational efforts required to solve the models. The studies discussed above used a variety of techniques to overcome 
these issues including: high-resolution simulation algorithms coupling upwind discretization and the Lax-Wendroff method, ${ }^{174,175,185,186}$ the method of classes, ${ }^{176-178,180,184}$ model reduction by means of a coordinate transformation method ${ }^{182}$ and other discretization methods such as the method of lines ${ }^{181}$ and the finite difference method. ${ }^{183}$ Multi-dimensional population balances appear also in the modeling of many particulate processes other than crystallization, such as granulation, polymerization and cell dynamics, which have led to new solution techniques for these numerically intensive models. Population balance discretization using a hierarchial two-tier solution strategy, ${ }^{187}$ adaptive high-resolution finite volume schemes ${ }^{188}$ and a discretization method based on a generalized moving pivot technique ${ }^{189}$ are just a few of the recent methods developed for solving multi-dimensional PBEs.

\subsection{Particle Size and Shape Characterization}

The measurement and characterization of particle size and shape distributions are important for implementing and validating the methods described above for determining PSDs. Several techniques and sensors are available that can measure particle size and/or PSDs (such as infrared spectroscopy and laser diffraction) but they generally cannot be used to measure particle shape directly. The most widely used technique today for simultaneous particle shape and size characterization is in-situ monitoring via video microscopy coupled with image analysis. Various in-situ monitoring tools currently in use include Lasentec's Focused Beam Reflectance Measurement ${ }^{190-193}$ (FBRM) which provides the chord length distribution (CLD) of the crystal population (many algorithms are currently available for transforming a CLD into a PSD), Lasentec's Process Vision and Measurement ${ }^{190,192-194}$ (PVM) that can take two-dimensional images of specific portions of the crystal population and the on-line imaging system by GlaxoSmithKline ${ }^{194-202}$ among other on-line and off-line imaging devices. These tools can be used in conjunction with conventional in-situ tech-

niques such as ATR-FTIR spectroscopy ${ }^{190,192,203}$ used for solution concentration measurements to measure supersaturation, and Raman spectroscopy ${ }^{192}$ for polymorph monitoring.

Commercial image analysis software is available but generally fails to successfully segment crystals from the image due to crystallizer hydrodynamics and continuous particle movement, ${ }^{204}$ thus motivating the need for improved on-line image analysis techniques. One method to evaluate crystal size and shape information from in situ crystallization images is by image segmentation 
(a)

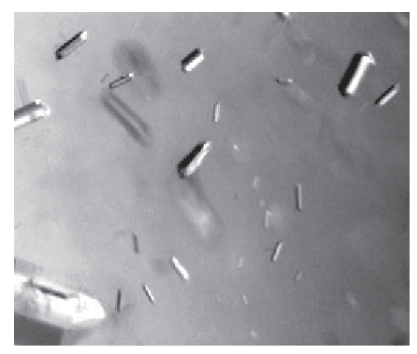

(c)

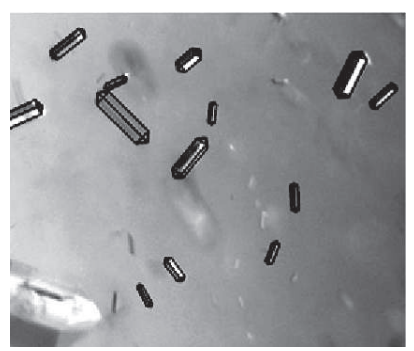

(b)

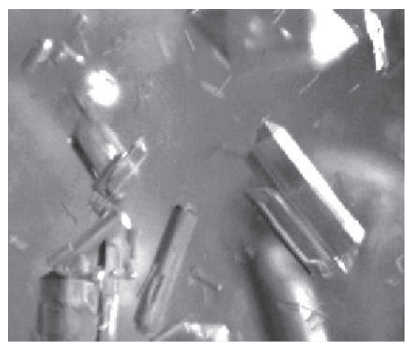

(d)

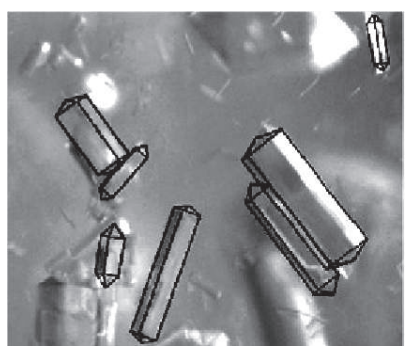

Figure 14: Image segmentation results for solutions of low solids concentration (Figures a and c) and high solids concentration (Figures b and d); reproduced from Larsen et al.. ${ }^{202}$

which separates crystals from the image background. Puel and co-workers have developed off-line imaging techniques to characterize crystal shape and size in two dimensions ${ }^{176,178,179}$ but these methods cannot be easily implemented in-situ. Calderon de Anda et al. ${ }^{194}$ presented a multi-scale segmentation method that takes into account the varied pixel intensity of on-line images (this article also provides a comprehensive review of other image segmentation techniques available at that time). Larsen et al. developed a commonly used image analysis algorithm using a model-based object recognition for needle-like particles ${ }^{201}$ and more recently extended the method to crystals of any shape that can be represented by a wire-frame model ${ }^{202}$; the authors were able to successfully implement these methods on solutions of moderate solids concentration. Figure 14 shows examples of the model-based shape recognition for crystals (M-SHARC) algorithm developed by Larsen et al. ${ }^{202}$ for solutions of low (Figure $14 \mathrm{a}$ and c) and high (Figure $14 \mathrm{~b}$ and d) solids concentration. Solutions of high solids density generally present a challenge to image segmentation methods ${ }^{205}$ due to the overlap of crystals and other phenomena such as agglomeration, breakage and attrition.

Once crystal images are segmented from the image background, size and shape descriptors are used to characterize possible crystal shapes. Recent work on shape descriptors include contour- 
based and region-based methods to determine aspect ratios, convexity, roundness, as well as other shape and size properties. A single shape descriptor is not sufficient to distinguish crystals of different shapes prompting the use of alternative shape descriptors. ${ }^{204,206-208}$ A recent review by Zhang and $\mathrm{Lu}^{209}$ discusses several shape descriptor techniques. Another commonly used technique is Fourier descriptors to transform contours and regions of particle images into shape characteristics. ${ }^{194,198,210}$ Most image segmentation and shape descriptor techniques provide 2D shape information whereas a recently developed camera model by Li et al. ${ }^{198}$ constructs 3-D shapes from 2-D images.

The on-line imaging and image analysis techniques discussed above can be used to estimate crystal face growth rates and kinetics. One approach is to model crystal growth and shape using multi-dimensional population balances (as discussed above) and estimate kinetic parameters from off-line images ${ }^{190}$; however, in order to implement these measurements into a control scheme, realtime growth kinetics are required. Puel and co-workers have measured in-situ growth kinetics of rodlike hydroquinone crystals ${ }^{178}$ and later developed a two-dimensional model to simulate the growth of platelet-crystals in batch seeded cooling crystallization to determine the kinetics of nucleation and attrition mechanisms. ${ }^{180}$ L-Glutamic acid has multiple polymorphs and the needle-like $\beta$ form has been the subject of several in situ imaging studies modeled by multi-dimensional population balances to estimate growth kinetics. ${ }^{192,199,200}$ Kempkes et al. ${ }^{193}$ presented a procedure to interpret in situ images to estimate growth rates of a population of cuboids. The experimental studies presented here are generally limited to measuring growth rates in two directions due to the $2 \mathrm{D}$ imaging techniques currently available; however, in the future with proper 3D imaging, growth rates of individual facets could be measured. ${ }^{211}$

\subsection{Shape Control}

Population balance models, coupled with on-line measurements can be used for developing modelpredictive algorithms. Such algorithms can provide insight into optimal control policies to produce crystals of a certain size and shape. Patience and Rawlings ${ }^{204}$ used in situ images from a cooling crystallizer to develop a feedback control algorithm using additive concentration as the manipulated variable to control crystal shape. They effectively added sodium dithionate to a solution of growing sodium chlorate crystals in order to change their shapes from cubic to tetrahedral while using the 
percentage of cubic crystals as the input to the feedback controller. In another study by Yang et al., ${ }^{212}$ the effects of different cooling modes were investigated for a batch cooling crystallization by using the supersaturation as the manipulated variable to control the shape of KDP crystals; this can be beneficial in selecting an optimal cooling profile for a desired product shape. Ultimately, if shape and size can be effectively controlled then there is also the possibility of controlling polymorphic transformations as demonstrated by the C-control strategies implemented by Hermanto et al. ${ }^{213}$ for the transformation of L-glutamic acid from the metastable $\alpha$ form to the stable $\beta$ form. Braatz and co-workers ${ }^{214}$ have recently reviewed the advances in the direct design and control of pharmaceutical crystallization processes and discuss the possible applications to more complicated tasks such as polymorph control and protein crystallization.

Through the use of multi-dimensional population balances models, in situ imaging techniques and real-time image analysis, model-predictive control schemes could potentially automatically control the size and shape of an entire crystal population in order to meet product specifications. For further insight into the techniques presented in this section the reader is referred to the following recent reviews. ${ }^{211,215,216}$

\section{Conclusions and Future Directions}

Significant progress has been made in the past several years developing engineering tools that allow for improved crystallization processing design. In particular, methods have been developed to predict steady-state crystal shapes through both molecular simulation and mechanistic modeling. Some of these methods provide the ability to account for the effects of single solvents as well as tailor-made additives on crystal shapes. The mechanistic modeling techniques can be performed fast enough (on the order of minutes of calculation time for current personal computers) that they have the potential to be implemented as part of product and process development. As we have shown through scaling arguments, the shapes of most molecular organic crystals growing by spirals are determined by the rates of surface integration on each face and not by bulk transport limitations. Thus, bulk transport effects in solution growth of molecular organic crystals are important only insofar as the local supersaturation is a function of position within the crystallizer. Further development continues for multi-dimensional population balances, including their solution techniques and implementation in real time feedback control. The combination of these modeling efforts with 
on-line shape measurement and image analysis techniques provides a method to measure real time growth kinetics, indicating a promising future for model predictive crystallization control.

In the future, continued research in these areas should allow for the prediction of: (1) crystal shapes as a function of supersaturation, (2) the appropriate dominant growth mechanism for a given set of processing conditions and (3) the effect of multiple solvents and various types of additives on crystal shape. These predictive capabilities will enable the process design stage of crystalline product development to include, for instance, both solubility and shape considerations in solvent selection. Furthermore, a priori shape predictions based on mechanistic growth models (described in $\S 5.1)$ will be developed for inorganic material as new techniques for modeling long range interactions and simultaneous reaction and crystallization are developed. Additionally, these shape predictions can be coupled with devices that track the growth rates of individual crystal faces, improving the ability to modify crystal shapes and PSD in real time during crystallizer operation.

Several aspects of crystal shape engineering will require the development of breakthrough technologies for the field to continue to advance. In order to couple polymorph and solvent selection, new (more reliable) methods are required for polymorph prediction. Currently, a gap exists in the knowledge of transitions between the growth mechanisms of nuclei (where the polymorphic structure is established) to those of faceted crystals. Filling this knowledge gap will require significant improvements in simulation and experimental techniques to handle the range of length and time scales involved. While the relative growth rates of crystal faces can be predicted for several systems, a major advance is necessary to predict absolute growth rates from first principles.

Finally, the broader implementation of crystal shape engineering as a practice in product and process design will require the development of computer aided design tools for polymorph selection and shape prediction that are integrated with thermodynamic calculations (e.g., solubilities and surface energies) and data bases (e.g., crystallography, solvent properties, etc.).

\section{Acknowledgements}

The authors are grateful for financial support provided by the National Science Foundation (Grant Nos. CTS-0554718 and CBET-0651711). Acknowledgment is also made to Merck and Co., the Donors of the American Chemical Society Petroleum Research Fund (Grant \#44172-AC10) and the International Fine Particle Research Institute (IFPRI) for partial support of this research. The 
work of A.R.B. was supported by graduate fellowships from the National Science Foundation and the UC Regents. The authors also thank Prof. Todd Squires for helpful discussions of transport phenomena.

\section{Supporting Information Statement}

Enclosed in the supporting information is a derivation of the bulk transport limited model and a description of the method used to obtain the scaling of the depletion boundary layer thickness. 


\section{References}

1. Yang, H. G.; Sun, C. H.; Qiao, S. Z.; Zou, J.; Liu, G.; Smith, S. C.; Cheng, H. M.; Lu, G. Q. Anatase $\mathrm{TiO}_{2}$ single crystals with a large percentage of reactive facets. Nature $\mathbf{2 0 0 8} 453,638$.

2. Variankaval, N.; Cote, A. S.; Doherty, M. F. From Form to Function: Crystallization of Active Pharmaceutical Ingredients. AIChE J. 2008 54, (to be published in the July issue).

3. Bauer, J.; Spanton, S.; Henry, R.; Quick, J.; Dziki, W.; Porter, W.; Morris, J. Ritonavir: An Extraordinary Example of Conformational Polymorphism. Pharm. Res. 2001 18, 859.

4. Bernstein, J. Polymorphism in Molecular Crystals. Clarendon Press: Oxford, 2002.

5. Yau, S.; Vekilov, P. Direct Observation of Nucleus Structure and Nucleation Pathways in Apoferritin Crystallization. J. Am. Chem. Soc. 2001 123, 1080.

6. Yu, L. Nucleation of One Polymorph by Another. J. Am. Chem. Soc. 2003 125, 6380.

7. Gibbs, J. W. The Scientific Papers of J. Willard Gibbs, vol. 1, Thermodynamics. Dover: New York, 1961.

8. Volmer, M.; A., W. Z. Phys. Chem. 1926 119, 227.

9. Farkas, Z. Z. Phys. Chem. 1927 125, 236.

10. Becker, R.; Doring, W. Ann. Phys. 1935 24, 719.

11. Kelton, K. F. Crystal Nucleation in Liquids and Glasses, vol. 45 of Solid State Physics. Academic Press, 1991.

12. Zettlemoyer, A. (ed.). Nucleation. Dekker, 1969.

13. Kashchiev, D. Nucleation: Basic Theory with Applications. Butterworth-Heinemann, 2000.

14. Wu, D.; Granasy, L.; Spaepen, F. Nucleation and the Solid-Liquid Interfacial Free Energy. MRS Bull. Dec 2004, 945.

15. Zykova-Timan, T.; Valeriani, C.; Sanz, E.; Frenkel, D.; Tosatti, E. Irreducible Finite-Size Effects in the Surface Free Energy of $\mathrm{NaCl}$ Crystals from Crystal-Nucleation Data. Phys. Rev. Lett. 2008 100, 036103. 
16. Leyssale, J.-M.; Delhommelle, J.; Millot, C. Hit and miss of classical nucleation theory as revealed by a molecular simulation study of crystal nucleation in supercooled sulfur hexafluoride. J. Chem. Phys. 2007 127, 044504.

17. Cacciuto, A.; Frenkel, D. Stresses Inside Critical Nuclei. J. Phys. Chem. B 2005 109, 6587.

18. Kashchiev, D.; Vekilov, P. G.; Kolomeisky, A. B. Kinetics of two-step nucleation of crystals. J. Chem. Phys. 2005 122, 244706.

19. ten Wolde, P. R.; Frenkel, D. Enhancement of Protein Crystal Nucleation by Critical Density Fluctuations. Science 1997 27r, 1975.

20. Lomakin, A.; Asherie, N.; Benedek, G. Liquid-solid Transition in Nuclei of Protein Crystals. Proc. Natl. Acad. Sci. U. S. A. 2003 100, 10254.

21. Soga, K. G.; Melrose, J. R.; Ball, R. C. Metastable states and the kinetics of colloid phase separation. J. Chem. Phys. 1999 110, 2280.

22. Sayle, T.; Catlow, C.; Maphanga, R.; Ngoepe, P.; Sayle, D. Generating $\mathrm{MnO}_{2}$ Nanoparticles Using Simulated Amorphization and Recrystallization. J. Am. Chem. Soc. 2005 127, 12828.

23. Vekilov, P. Dense Liquid Precursor for the Nucleation of Ordered Solid Phases from Solution. Cryst. Growth Des. 2004 4, 671.

24. Pan, W.; Kolomeisky, A. B.; Vekilov, P. G. Nucleation of ordered solid phases of proteins via a disordered high-density state: Phenomenological approach. J. Chem. Phys. 2005 122, 174905 .

25. Zhang, T.; Liu, X. How Does a Transient Amorphous Precursor Template Crystallization. J. Am. Chem. Soc. 2007 129, 13520.

26. Valeriani, C.; Sanz, E.; Frenkel, D. Rate of homogeneous crystal nucleation in molten NaCl. J. Chem. Phys. 2005 122, 194501.

27. Gasser, U.; Weeks, E.; Schofield, A.; Pusey, P.; Weitz, D. Real-Space Imaging of Nucleation and Growth in Colloidal Crystallization. Science 2001 292, 258. 
28. Balsara, N. P.; Rappl, T. J.; Lefebvre, A. A. Does conventional nucleation occur during phase separation in polymer blends? J. Polym. Sci., Part B: Polym. Phys. 2004 42, 1793.

29. Pino-Garcia, O.; Rasmuson, A. Influence of Additives on Nucleation of Vanillin: Experiments and Introductory Molecular Simulations. Cryst. Growth Des. 2004 4, 1025.

30. Roelands, C.; Roestenberg, R.; terHorst, J.; Kramer, H.; Jansens, P. Development of an Experimental Method to Measure Nucleation Rates in Reactive Precipitation. Cryst. Growth Des. 2004 4, 921.

31. Towler, C.; Davey, R.; Lancaster, R.; Price, C. Impact of Molecular Speciation on Crystal Nucleation in Polymorphic Systems. J. Am. Chem. Soc. 2004 126, 13347.

32. Weissbuch, I.; Lahav, M.; Leiserowitz, L. Toward Stereochemical Control, Monitoring, and Understanding of Crystal Nucleation. Cryst. Growth Des. 2003 3, 125.

33. Meijer, E.; Frenkel, D. Melting Line of Yukawa System by Computer Simulation. J. Chem. Phys. 1991 94, 2269.

34. O’Malley, B.; Snook, I. Crystal Nucleation in the Hard Sphere System. Phys. Rev. Lett. 2003 90, 085702 .

35. Anwar, J.; Boateng, P. Computer Simulation of Crystallization from Solution. J. Am. Chem. Soc. 1998 120, 9600.

36. Auer, S.; Frenkel, D. Numerical prediction of absolute crystallization rates in hard-sphere colloids. J. Chem. Phys. 2004 120, 3015.

37. Trudu, F.; Donadio, D.; Parrinello, M. Freezing of a Lennard-Jones Fluid: From Nucleation to Spinodal Regime. Phys. Rev. Lett. 2006 97, 105701.

38. Desgranges, C.; Delhommelle, J. Polymorph selection during the crystallization of Yukawa systems. J. Chem. Phys. 2007 126, 054501.

39. Browning, A. R.; Doherty, M. F.; Fredrickson, G. H. Nucleation and polymorph selection in a model colloidal fluid. Phys. Rev. E: Stat., Nonlinear, Soft Matter Phys. 2008 77, 041604. 
40. Izumi, S.; Hara, S.; Kumagai, T.; Sakai, S. Molecular dynamics study of homogeneous crystal nucleation in amorphous silicon. J. Cryst. Growth 2005 274, 47.

41. Leyssale, J. M.; Delhommelle, J.; Millot, C. A molecular dynamics study of homogeneous crystal nucleation in liquid nitrogen. Chem. Phys. Lett. 2003 375, 612.

42. Zahn, D. Atomistic Mechanism of $\mathrm{NaCl}$ Nucleation from an Aqueous Solution. Phys. Rev. Lett. 2004 92, 040801.

43. Gavezzotti, A. Molecular Aggregation of Acetic Acid in a Carbon Tetrachloride Solution: A Molecular Dynamics Study with a View to Crystal Nucleation. Chem. Eur. J. 1999 5, 567.

44. Beckham, G.; Peters, B.; Starbuck, C.; Variankaval, N.; Trout, B. Surface-Mediated Nucleation in the Solid-State Polymorph Transformation of Terephthalic Acid. J. Am. Chem. Soc. 2007 129, 4714.

45. Peters, B.; Trout, B. L. Obtaining reaction coordinates by likelihood maximization. J. Chem. Phys. 2006 125, 054108.

46. Wulff, G. Z. Kryst. 1901 34, 449.

47. Herring, C. Some Theorems on the Free Energies of Crystal Surfaces. Phys. Rev. 1951 82, 87.

48. Mutaftschiev, B. Nucleation Theory. In Handbook of Crystal Growth, 1a FundamentalsThermodynamics and Kinetics, D. T. J. Hurle, ed., chap. 4. North-Holland: Amsterdam, 1993, $187-247$.

49. Kern, R. The Equilibrium Form of a Crystal. In Morphology of Crystals: Part A, I. Sunagawa, ed., chap. 2. Terra Scientific Publishing Company: Tokyo, 1987, 77-206.

50. Frank, F. C. On the Kinematic Theory of Crystal Growth and Dissolution Processes. In Growth and Perfection of Crystals, R. H. Doremus; B. W. Roberts; D. Turnbull, eds. Wiley: New York, 1958.

51. Chernov, A. A. The Kinetics of the Growth Froms of Crystals. Sov. Phys. Cryst. 1963 7, 728. 
52. Zhang, Y.; Sizemore, J. P.; Doherty, M. F. Shape evolution of 3-dimensional faceted crystals. AIChE J. 2006 52, 1906.

53. Snyder, R. C.; Doherty, M. F. Faceted crystal shape evolution during dissolution or growth. AIChE J. 2007 53, 1337.

54. Bravais, A. Études Crystallographiques. Gauthier-Villars: Paris, 1866.

55. Friedel, M. G. Études Sur la loi de Bravais. Bull. Soc. Franc. Miner. 1907 9, 326.

56. Donnay, J. D. H.; Harker, D. A New Law of Crystal Morphology Extending the Law of Bravais. Amer. Min. 1937 22, 446.

57. Hartman, P.; Perdok, W. G. On the relations between structure and morphology of crystals. I. Acta Crystallogr. 1955 8, 49.

58. Hartman, P.; Perdok, W. G. On the relations between structure and morphology of crystals. II. Acta Crystallogr. 1955 8, 521.

59. Clydesdale, G.; Docherty, R.; Roberts, K. J. HABIT - a program for predicting the morphology of molecular crystals. Comput. Phys. Commun. 1991 64, 311.

60. Grimbergen, R. F. P.; Meekes, H.; Bennema, P.; Strom, C. S.; Vogels, L. J. P. On the Prediction of Crystal Morphology. I. The Hartman-Perdok Theory Revisited. Acta Crystallogr., Sect. A: Found. Crystallogr. 1998 54, 491.

61. Hartman, P.; Bennema, P. The attachment energy as a habit controlling factor : I. Theoretical considerations. J. Cryst. Growth 1980 49, 145.

62. Kossel, W. Nachur. Ges. Gottingen 1927 206, 135.

63. Stranski, I. N. Z. Phys. Chem. 1928 136, 259.

64. Kuroda, T. Growth of a crystal surface with non-uniformity in supersaturation due to laminar flow of solution along the surface. J. Cryst. Growth 1985 71, 84.

65. Wilcox, W. R. Transport phenomena in crystal growth from solution. Prog. Cryst. Growth Charact. Mater. 1993 26, 153. 
66. Land, T. A.; De Yoreo, J. J. The evolution of growth modes and activity of growth sources on canavalin investigated by in situ atomic force microscopy. J. Cryst. Growth 2000 208, 623.

67. Chernov, A. A. Modern Crystallography III. Crystal Growth. Springer-Verlag: Berlin, 1984.

68. Frenkel, J. On the surface motion of particles in crystals and the natural roughness of crystalline faces. J. Phys. U.S.S.R. 1945 9, 392.

69. Burton, W. K.; Cabrera, N.; Frank, F. C. The Growth of Crystals and the Equilibrium Structure of their Surfaces. Phil. Trans. Roy. Soc. A 1951 243, 299.

70. Zhang, J.; Nancollas, G. H. Kink densities along a crystal surface step at low temperatures and under nonequilibrium conditions. J. Cryst. Growth 1990 106, 181.

71. Cuppen, H. M.; Meekes, H.; van Veenendaal, E.; van Enckevort, W. J. P.; Bennema, P.; Reedijk, M. F.; Arsic, J.; Vlieg, E. Kink density and propagation velocity of the $\left[\begin{array}{lll}0 & 1 & 0\end{array}\right]$ step on the Kossel (1 0 0 $)$ ) surface. Surf. Sci. 2002 506, 183.

72. Cuppen, H. M.; Meekes, H.; van Enckevort, W. J. P.; Vlieg, E.; Knops, H. J. F. Nonequilibrium free energy and kinetic roughening of steps on the Kossel(001) surface. Phys. Rev. B: Condens. Matter Mater. Phys. 2004 69, 245404.

73. Volmer, M. Z. Physik. 1922 9, 193.

74. Ohara, M.; Reid, R. C. Modeling Crystal Growth Rates from Solution. Prentice-Hall, Inc.: New Jersey, 1973.

75. Winn, D.; Doherty, M. F. A new technique for predicting the shape of solution-grown organic crystals. AIChE J. 1998 44, 2501.

76. Cuppen, H.; Meekes, H.; van Enckevort, W.; Vlieg, E. Birth-and-spread growth on the Kossel and a non-Kossel surface. J. Cryst. Growth 2006 286, 188.

77. De Yoreo, J. J.; Vekilov, P. G. Principles of Crystal Nucleation and Growth. In Biomineralization, P. M. Dove; J. J. De Yoreo; S. Weiner, eds., vol. 54 of Reviews in Mineralogy 6 Geochemistry. Mineralogical Society of America: Washington, DC, 2003. 
78. Volmer, M.; Schultze, W. Z. Phys. Chem. (A) 1931 156, 1.

79. Frank, F. C. The influence of dislocations on crystal growth. Disc. Faraday Soc. 1949 5, 48.

80. Snyder, R. C.; Doherty, M. F. Predicting Crystal Growth by Spiral Motion. Proceedings of the Royal Society A 2008, Submitted.

81. Markov, I. V. Crystal Growth for Beginners, Fundamentals of Nucleation, Crystal Growth and Epitaxy. World Scientific: Singapore, 2003.

82. Winn, D.; Doherty, M. F. Modeling crystal shapes of organic materials grown from solution. AIChE J. 2000 46, 1348.

83. Liu, X. Y.; Boek, E. S.; Briels, W. J.; Bennema, P. Prediction of crystal growth morphology based on structural analysis of the solid-fluid interface. Nature 1995 374, 342.

84. Winn, D.; Doherty, M. F. Predicting the shape of organic crystals grown from polar solvents. Chem. Eng. Sci. 2002 57, 1805.

85. Shekunov, B.; Grant, D. In Situ Optical Interferometric Studies of the Growth and Dissolution Behavior of Paracetamol (Acetaminophen). 1. Growth Kinetics. J. Phys. Chem. B 1997101 , 3973.

86. Ristic, R.; Finnie, S.; Sheen, D.; Sherwood, J. Macro- and Micromorphology of Monoclinic Paracetamol Grown from Pure Aqueous Solution. J. Phys. Chem. B 2001 105, 9057.

87. Vekilov, P.; Alexander, J. Dynamics of Layer Growth in Protein Crystallization. Chem. Rev. $2000100,2061$.

88. Voronkov, V. V. Dislocation mechanism of growth with a low kink density. Sov. Phys. Cryst. $197318,19$.

89. Teng, H. H.; Dove, P. M.; Orme, C. A.; De Yoreo, J. J. Thermodynamics of Calcite Growth: Baseline for Understanding Biomineral Formation. Science 1998 282, 724.

90. Chernov, A. A. Crystal growth science between the centuries. J. Mater. Sci.: Mater. Electron. 2001 12, 437. 
91. Chernov, A. A. Notes on interface growth kinetics 50 years after Burton, Cabrera and Frank. J. Cryst. Growth 2004 264, 499.

92. Rashkovich, L.; Gvozdev, N.; Sil'nikova, M.; Chernov, A. Fluctuations in the step velocity and the generation of a dislocation spiral on the (101) face of monoclinic lysozyme crystals. Crystallogr. Rep. 2002 47, 859.

93. Rashkovich, L.; Petrova, E.; Shustin, O.; Chernevich, T. Formation of a dislocation spiral on the (010) face of a potassium hydrogen phthalate crystal. Phys. Solid State 2003 45, 400.

94. Cabrera, N.; Levine, M. M. XLV. On the dislocation theory of evaporation of crystals. Philos. Mag. 1956 1, 450.

95. Gilmer, G. H.; Bennema, P. Simulation of Crystal Growth with Surface Diffusion. J. Appl. Phys. 1972 43, 1347.

96. Rohl, A. L. Computer prediction of crystal morphology. Curr. Opin. Solid State Mater. Sci. $20037,21$.

97. van der Eerden, J. P. J. M. Molecular simulations of crystal growth processes. In Crystal Growth - From Fundamentals To Technology, G. Müller; J. J. Métois; P. Rudolph, eds. Elsevier: Amsterdam, 2004, 187-213.

98. Boerrigter, S.; Josten, G.; vandeStreek, J.; Hollander, F.; Los, J.; Cuppen, H.; Bennema, P.; Meekes, H. MONTY: Monte Carlo Crystal Growth on Any Crystal Structure in Any Crystallographic Orientation; Application to Fats. J. Phys. Chem. A 2004 108, 5894.

99. Zepeda-Ruiz, L. A.; Maiti, A.; Gee, R.; Gilmer, G. H.; Weeks, B. L. Size and habit evolution of PETN crystals-a lattice Monte Carlo study. J. Cryst. Growth 2006 291, 461.

100. Piana, S.; Reyhani, M.; Gale, J. D. Simulating micrometre-scale crystal growth from solution. Nature 2005 438, 70.

101. Anderson, M. W.; Agger, J. R.; Meza, L. I.; Chong, C. B.; Cundy, C. S. Crystal growth in nanoporous framework materials. Faraday Discuss. 2007 136, 143. 
102. Rodrguez-hornedo, N.; Murphy, D. Significance of controlling crystallization mechanisms and kinetics in pharmaceutical systems. J. Pharm. Sci. 1999 88, 651.

103. Mehta, S. C.; Bernardo, P. D.; Higuchi, W. I.; Simonelli, A. P. Rate of crystal growth of sulfathiazole and methylprednisolone. J. Pharm. Sci. 1970 59, 638.

104. Kind, M.; Mersmann, A. On supersaturation during mass crystallization from solution. Chem. Eng. Technol. 1990 13, 50.

105. Li, L.; Rodriguez-Hornedo, N. Growth kinetics and mechanism of glycine crystals. J. Cryst. Growth 1992 121, 33.

106. Veesler, S.; Lafferrere, L.; Garcia, E.; Hoff, C. Phase Transitions in Supersaturated Drug Solution. Org. Process Res. Dev. 2003 7, 983.

107. Onuma, K.; Kameyama, T.; Tsukamoto, K. In situ study of surface phenomena by real time phase shift interferometry. J. Cryst. Growth 1994 137, 610.

108. McLoughlin, M. J.; Mays, T. J.; Price, R. Growth Spiral Activity and Step Velocities on a Crystal Surface. Phys. Rev. Lett. 2005 95, 115504.

109. Rosenberger, F. Protein crystallization. J. Cryst. Growth 1996 166, 40.

110. Vekilov, P. G.; Ataka, M.; Katsura, T. Growth process of protein crystals revealed by laser Michelson interferometry investigation. Acta Crystallogr., Sect. D: Biol. Crystallogr. 1995 $51,207$.

111. Chernov, A. A. Formation of crystals in solutions. Contemporary Physics 1989 30, 251.

112. Rashkovich, L. N.; Shekunov, B. Y. Morphology of growing vicinal surface; prismatic faces of ADP and KDP crystals in solutions. J. Cryst. Growth 1990 100, 133.

113. Bazzana, S.; Dumrul, S.; Warzywoda, J.; Hsiao, L.; Klass, L.; Knapp, M.; Rains, J. A.; Stein, E. M.; Sullivan, M. J.; West, C. M.; Woo, J. Y.; Sacco, A., Jr. Observations of layer growth in synthetic zeolites by field emission scanning electron microscopy. Studies in Surface Science and Catalysis 2002 142, 117. 
114. Dumrul, S.; Bazzana, S.; Warzywoda, J.; Biederman, R. R.; Sacco, A. Imaging of crystal growth-induced fine surface features in zeolite A by atomic force microscopy. Microporous Mesoporous Mater. 2002 54, 79.

115. Yilmaz, B.; Miraglia, P. Q.; Warzywoda, J.; Sacco, A. Synthesis of titanosilicate ETS-4 with controlled morphology and investigation of its crystallization kinetics. Microporous Mesoporous Mater. 2004 71, 167.

116. Miraglia, P. Q.; Yilmaz, B.; Warzywoda, J.; Sacco, A., Jr. Surface growth mechanisms and structural faulting in the growth of large single and spherulitic titanosilicate ETS-4 crystals. J. Cryst. Growth 2004 270, 674.

117. Hillner, P. E.; Gratz, A. J.; Manne, S.; Hansma, P. K. Atomic-scale imaging of calcite growth and dissolution in real time. Geology 1992 20,359.

118. Teng, H. H.; Dove, P. M.; De Yoreo, J. J. Kinetics of calcite growth: surface processes and relationships to macroscopic rate laws. Geochim. Cosmochim. Acta 2000 64, 2255.

119. De Yoreo, J. J.; Burnham, A. K.; Whitman, P. K. Developing $\mathrm{KH}_{2} \mathrm{PO}_{4}$ and $\mathrm{KD}_{2} \mathrm{PO}_{4}$ crystals for the world's most powerful laser. Int. Mat. Rev. 2002 47, 113.

120. Thomas, T. N.; Land, T. A.; Martin, T.; Casey, W. H.; DeYoreo, J. J. AFM investigation of step kinetics and hillock morphology of the 100 face of KDP. J. Cryst. Growth 2004260 , 566.

121. Qiu, S. R.; Wierzbicki, A.; Orme, C. A.; Cody, A. M.; Hoyer, J. R.; Nancollas, G. H.; Zepeda, S.; De Yoreo, J. J. From the Cover: Molecular modulation of calcium oxalate crystallization by osteopontin and citrate. PNAS 2004 101, 1811.

122. Touryan, L. A.; Lochhead, M. J.; Marquardt, B. J.; Vogel, V. Sequential switch of biomineral crystal morphology using trivalent ions. Nat. Mater. 2004 3, 239.

123. Jung, T.; Sheng, X. X.; Choi, C. K.; Kim, W. S.; Wesson, J. A.; Ward, M. D. Probing crystallization of calcium oxalate monohydrate and the role of macromolecule additives with in situ atomic force microscopy. Langmuir 2004 20, 8587. 
124. Weisenhorn, A. L.; Dougall, J. E. M.; Gould, S. A. C.; Cox, S. D.; Wise, W. S.; Massie, J.; Maivald, P.; Elings, V. B.; Stucky, G. D.; Hansma, P. K. Imaging and Manipulating Molecules on a Zeolite Surface with an Atomic Force Microscope. Science 1990 247, 1330.

125. Agger, J.; Pervaiz, N.; Cheetham, A.; Anderson, M. Crystallization in Zeolite A Studied by Atomic Force Microscopy. J. Am. Chem. Soc. 1998 120, 10754.

126. Anderson, M. W.; Ohsuna, T.; Sakamoto, Y.; Liu, Z.; Carlsson, A.; Terasaki, O. Modern microscopy methods for the structural study of porous materials. Chem. Commun. 2004, 907.

127. Wakihara, T.; Sugiyama, A.; Okubo, T. Crystal growth of faujasite observed by atomic force microscopy. Microporous Mesoporous Mater. 2004 70, 7.

128. Durbin, S. D.; Carlson, W. E.; Saros, M. T. In situ studies of protein crystal growth by atomic force microscopy. J. Phys. D: Appl. Phys. 1993 26, B128.

129. Yip, C. M.; Ward, M. D. Atomic force microscopy of insulin single crystals: direct visualization of molecules and crystal growth. Biophys. J. 1996 71, 1071.

130. McPherson, A.; Malkin, A. J.; Kuznetsov, Y. G. Atomic force microscopy in the study of macromolecular crystal growth. Annu. Rev. Biophys. Biomol. Struct. 2000 29, 361.

131. Thompson, C.; Davies, M. C.; Roberts, C. J.; Tendler, S. J. B.; Wilkinson, M. J. The effects of additives on the growth and morphology of paracetamol (acetaminophen) crystals. Int. J. Pharm. 2004 280, 137.

132. Keel, T.; Thompson, C.; Davies, M.; Tendler, S.; Roberts, C. AFM studies of the crystallization and habit modification of an excipient material, adipic acid. Int. J. Pharm. 2004 280, 185.

133. Sours, R.; Zellelow, A.; Swift, J. An in Situ Atomic Force Microscopy Study of Uric Acid Crystal Growth. J. Phys. Chem. B 2005 109, 9989.

134. Stoica, C.; van Enckevort, W.; Meekes, H.; Vlieg, E. Interlaced spiral growth and step splitting on a steroid crystal. J. Cryst. Growth 2007 299, 322. 
135. Chernov, A. A.; Rashkovich, L. N.; Yaminski, I. V.; Gvozdev, N. V. Kink kinetics, exchange fluxes, 1D 'nucleation' and adsorption on the (010) face of orthorhombic lysozyme crystals. J. Phys.: Condens. Matter 1999 11, 9969.

136. Chernov, A.; Rashkovich, L.; Vekilov, P. Steps in solution growth: dynamics of kinks, bunching and turbulence. J. Cryst. Growth 2005 275, 1.

137. Rashkovich, L.; De Yoreo, J.; Orme, C.; Chernov, A. In situ atomic force microscopy of layer-by-layer crystal growth and key growth concepts. Crystallogr. Rep. 2006 51, 1063.

138. Georgiou, D. K.; Vekilov, P. G. A fast response mechanism for insulin storage in crystals may involve kink generation by association of 2D clusters. PNAS 2006 103, 1681.

139. Davis, K. J.; Dove, P. M.; De Yoreo, J. J. The Role of $\mathrm{Mg}^{2+}$ as an Impurity in Calcite Growth. Science 2000 290, 1134.

140. Danesh, A.; Chen, X.; Davies, M.; Roberts, C.; Sanders, G.; Tendler, S.; Williams, P.; Wilkins, M. Polymorphic Discrimination Using Atomic Force Microscopy: Distinguishing between Two Polymorphs of the Drug Cimetidine. Langmuir 2000 16, 866.

141. Wells, A. F. Crystal Habit and Internal Structure-I. Philos. Mag. 1946 37, 184.

142. Wells, A. Abnormal and Modified Crystal Growth. Introductory Paper. Discuss. Faraday Soc. 1949 5, 197201.

143. Davey, R. J.; Milisavljevic, B.; Bourne, J. R. Solvent interactions at crystal surfaces: the kinetic story of .alpha.-resorcinol. J. Phys. Chem. 1988 92, 2032.

144. Davey, R. J.; Mullin, J. W.; Whiting, M. J. L. Habit Modification Of Succinic Acid Crystals Grown From Different Solvents. J. Cryst. Growth 1982 58, 304.

145. Gordon, R. E.; Amin, S. I. Crystallization of Ibuprofen. United States Patent Number 4,476,248, 1984.

146. Davey, R. J. The role of the solvent in crystal growth from solution. J. Cryst. Growth 1986 $76,637$. 
147. Lahav, M.; Leiserowitz, L. The effect of solvent on crystal growth and morphology. Chem. Eng. Sci. 2001 56, 2245.

148. Lechuga-Ballesteros, D.; Rodriguez-Hornedo, N. The Influence Of Additives On The GrowthKinetics And Mechanism Of L-Alanine Crystals. Int. J. Pharm. 1995 115, 139.

149. Davey, R. J.; Black, S. N.; Logan, D.; Maginn, S. J.; Fairbrother, J. E.; Grant, D. J. W. Structural And Kinetic Features Of Crystal-Growth Inhibition - Adipic Acid Growing In The Presence Of N-Alkanoic Acids. J. Chem. Soc., Faraday Trans. 1992 88, 3461.

150. Garti, N.; Zour, H. The effect of surfactants on the crystallization and polymorphic transformation of glutamic acid. J. Cryst. Growth 1997 172, 486.

151. Hendriksen, B. A.; Grant, D. J. W.; Meenan, P.; Green, D. A. Crystallisation of paracetamol (acetaminophen) in the presence of structurally related substances. J. Cryst. Growth 1998 183,629 .

152. Weissbuch, I.; Addadi, L.; Berkovitch-Yellin, Z.; Gati, E.; Weinstein, S.; Lahav, M.; Leiserowitz, L. Centrosymmetric Crystals For The Direct Assignment Of The AbsoluteConfiguration Of Chiral Molecules - Application To The Alpha-Amino-Acids By Their Effect On Glycine Crystals. J. Am. Chem. Soc. 1983 105, 6615.

153. Michaels, A. S.; Tausch, F. W. Modification Of Growth Rate And Habit Of Adipic Acid Crystals With Surfactants. J. Phys. Chem. 1961 65, 1730.

154. Klug, D. L.; van Mil, J. H. Purification of Adipic Acid. U.S. Patent 5,296,639, 1994.

155. Michaels, A. S.; Colville, A. R. The effect of surface active agents on crystal growth rate and crystal habit. J. Phys. Chem. 1960 64, 13.

156. DeVries, A. L. Glycoproteins As Biological Antifreeze Agents In Antarctic Fishes. Science 1971 172, 1152.

157. Raymond, J. A.; DeVries, A. L. Adsorption Inhibition As A Mechanism Of Freezing Resistance In Polar Fishes. Proc. Natl. Acad. Sci. U. S. A. 1977 74, 2589. 
158. Cabrera, N.; Vermilyea, D. The Growth of Crystals from Solution. In Growth and Perfection of Crystals, R. Doremus; B. Roberts; D. Turnbull, eds. John Wiley \& Sons, Inc.: New York, 1958.

159. Land, T.; Martin, T.; Potapenko, S.; Palmore, G.; DeYoreo, J. Recovery of surfaces from impurity poisoning during crystal growth. Nature 1999 399, 442.

160. Chernov, A. A. The spiral growth of crystals. Soviet Phys.-Usp. 1961 4, 116.

161. Cabrera, N.; Coleman, R. V. Theory of Crystal Growth from the Vapor. In The Art and Science of Growing Crystals, J. J. Gilman, ed. Wiley: New York, 1963.

162. Clydesdale, G.; Roberts, K. J.; Docherty, R. Modeling The Morphology Of Molecular-Crystals In The Presence Of Disruptive Tailor-Made Additives. J. Cryst. Growth 1994 135, 331.

163. Clydesdale, G.; Roberts, K. J.; Lewitas, K.; Docherty, R. Modeling The Morphology Of Molecular-Crystals In The Presence Of Blocking Tailor-Made Additives. J. Cryst. Growth $1994141,443$.

164. Hartman, P. Structure and Morphology. In Crystal Growth: An Introduction, P. Hartman, ed. North-Holland Publishing Co.: Amsterdam, 1973, 367-402.

165. Berkovitch-Yellin, Z. Toward an ab initio derivation of crystal morphology. J. Am. Chem. Soc. 1985 10\%, 8239.

166. Lighthill, M. J.; Whitham, G. B. On Kinematic Waves .1. Flood Movement In Long Rivers. Proc. Roy. Soc. London Ser. A 1955 229, 281.

167. Lighthill, M. J.; Whitham, G. B. On Kinematic Waves .2. A Theory Of Traffic Flow On Long Crowded Roads. Proc. Roy. Soc. London Ser. A 1955 229, 317.

168. Hamaguchi, S.; Dalvie, M. Microprofile Simulations For Plasma-Etching With Surface Passivation. J. Vac. Sci. Technol., A 1994 12, 2745.

169. Shankland, N.; Florence, A. J.; Cox, P. J.; Sheen, D. B.; Love, S. W.; Stewart, N. S.; Wilson, C. C. Crystal morphology of ibuprofen predicted from single-crystal pulsed neutron diffraction data. Chem. Commun. $1996,855$. 
170. Cano, H.; Gabas, N.; Canselier, J. Experimental study on the ibuprofen crystal growth morphology in solution. J. Cryst. Growth 2001 224, 335.

171. Randolph, A. D.; Larson, M. A. Theory of Particulate Processes: Analysis and Techniques of Continuous Crystallization. Academic Press: New York, 1988.

172. Ramkrishna, D. Population balances-theory and applications to particulate systems in engineering. Academic Press: San Diego, 2000.

173. Iveson, S. M. Limitations of one-dimensional population balance models of wet granulation processes. Powder Technol. 2002 124, 219.

174. Ma, D. L.; Braatz, R. D.; Tafti, D. K. Compartmental modeling of multidimensional crystallization. Internat. J. Modern Phys. B 2002 16, 383.

175. Ma, D. L.; Tafti, D. K.; Braatz, R. D. Optimal control and simulation of multidimensional crystallization processes. Comput. Chem. Eng. 2002 26, 1103.

176. Puel, F.; Marchal, P.; Klein, J. Habit transient analysis in industrial crystallization using two dimensional crystal sizing technique. Chem. Eng. Res. Des. 1997 75, 193.

177. Puel, F.; Fevotte, G.; Klein, J. P. Simulation and analysis of industrial crystallization processes through multidimensional population balance equations. Part 1: a resolution algorithm based on the method of classes. Chem. Eng. Sci. 2003 58, 3715.

178. Puel, F.; Fevotte, G.; Klein, J. P. Simulation and analysis of industrial crystallization processes through multidimensional population balance equations. Part 2: a study of semi-batch crystallization. Chem. Eng. Sci. 2003 58, 3729.

179. Oullion, M.; Puel, F.; Fevotte, G.; Righini, S.; Carvin, P. Industrial batch crystallization of a plate-like organic product. In situ monitoring and 2D-CSD modelling: Part 1: Experimental study. Chem. Eng. Sci. 2007 62, 820.

180. Oullion, M.; Puel, F.; Fevotte, G.; Righini, S.; Carvin, P. Industrial batch crystallization of a plate-like organic product. In situ monitoring and 2D-CSD modelling. Part 2: Kinetic modelling and identification. Chem. Eng. Sci. 2007 62, 833. 
181. Gerstlauer, A.; Mitrovic, A.; Motz, S.; Gilles, E. D. A population model for crystallization processes using two independent particle properties. Chem. Eng. Sci. 2001 56, 2553.

182. Briesen, H. Simulation of crystal size and shape by means of a reduced two-dimensional population balance model. Chem. Eng. Sci. 2006 61, 104.

183. Zhang, Y.; Doherty, M. F. Simultaneous prediction of crystal shape and size for solution crystallization. AIChE J. 2004 50, 2101.

184. Ma, C. Y.; Wang, X. Z.; Roberts, K. J. Morphological population balance for modeling crystal growth in face directions. AIChE J. 2008 54, 209.

185. Ma, D.; Tafti, D.; Braatz, R. High-Resolution Simulation of Multidimensional Crystal Growth. Ind. Eng. Chem. Res. 2002 41, 6217.

186. Gunawan, R.; Fusman, I.; Braatz, R. D. High resolution algorithms for multidimensional population balance equations. AIChE J. $\mathbf{2 0 0 4} 50,2738$.

187. Pinto, M. A.; Immanuel, C. D.; Doyle III, F. J. A feasible solution technique for higherdimensional population balance models. Comput. Chem. Eng. 2007 31, 1242.

188. Qamar, S.; Ashfaq, A.; Warnecke, G.; Angelov, I.; Elsner, M.; Seidel-Morgenstern, A. Adaptive high-resolution schemes for multidimensional population balances in crystallization processes. Comput. Chem. Eng. 2007 31, 1296.

189. Chakraborty, J.; Kumar, S. A new framework for solution of multidimensional population balance equations. Chem. Eng. Sci. 2007 62, 4112.

190. Gunawan, R.; Ma, D. L.; Braatz, M. F. R. D. Identification of kinetic parameters in multidimensional crystallization processes. Internat. J. Modern Phys. B 2002 16, 367.

191. Ruf, A.; Worlitschek, J.; Mazzotti, M. Modeling and Experimental Analysis of PSD Measurements through FBRM. Part. Part. Syst. Char. 2000 17, 167.

192. Scholl, J.; Bonalumi, D.; Vicum, L.; Mazzotti, M.; Muller, M. In Situ Monitoring and Modeling of the Solvent-Mediated Polymorphic Transformation of ifont size="-1" ¿ $\mathrm{L}_{\mathrm{i}} /$ font $_{\mathrm{i}}$ Glutamic Acid. Cryst. Growth Des. 2006 6, 881. 
193. Kempkes, M.; Eggers, J.; Mazzotti, M. Measurement of particle size and shape by FBRM and in situ microscopy. Chem. Eng. Sci. 2007 In Press, Corrected Proof, .

194. Calderon De Anda, J.; Wang, X.; Roberts, K. Multi-scale segmentation image analysis for the in-process monitoring of particle shape with batch crystallisers. Chem. Eng. Sci. 2005 $60,1053$.

195. Calderon De Anda, J.; Wang, X. Z.; Lai, X.; Roberts, K. J.; Jennings, K. H.; Wilkinson, M. J.; Watson, D.; Roberts, D. Real-time product morphology monitoring in crystallization using imaging technique. AIChE J. 2005 51, 1406.

196. Calderon De Anda, J.; Wang, X.; Lai, X.; Roberts, K. Classifying organic crystals via inprocess image analysis and the use of monitoring charts to follow polymorphic and morphological changes. J. Process Control 2005 15, 785.

197. Wang, X. Z.; Calderon De Anda, J.; Roberts, K. J.; Li, R. F.; Thomson, G. B.; White, G. Advances in on-line monitoring and control of the morphological and polymorphic forms of organic crystals grown from solution. Kona 2005 23, 69.

198. Li, R. F.; Thomson, G. B.; White, G.; Wang, X. Z.; Calderon De Anda, J.; Roberts, K. J. Integration of crystal morphology modeling and on-line shape measurement. AIChE J. 2006 52, 2297.

199. Ma, C.; Wang, X.; Roberts, K. Multi-dimensional population balance modeling of the growth of rod-like 1 -glutamic acid crystals using growth rates estimated from in-process imaging. Adv. Powder Technol. 2007 18, 707.

200. Wang, X. Y.; Calderon De Anda, J.; Roberts, K. J. Real-time measurement of the growth rates of individual crystal facets using imaging and image analysis - A feasibility study on needle-shaped crystals of L-glutamic acid. Chem. Eng. Res. Des. 2007 85, 921.

201. Larsen, P.; Rawlings, J.; Ferrier, N. An algorithm for analyzing noisy, in situ images of high-aspect-ratio crystals to monitor particle size distribution. Chem. Eng. Sci. 2006 61, 5236. 
202. Larsen, P.; Rawlings, J.; Ferrier, N. Model-based object recognition to measure crystal size and shape distributions from in situ video images. Chem. Eng. Sci. 2007 62, 1430.

203. Pollanen, K.; Hakkinen, A.; Reinikainen, S. P.; Louhi-Kultanen, M.; Nystrom, L. A study on batch cooling crystallization of sulphathiazole - Process monitoring using ATR-FTIR and product characterization by automated image analysis. Chem. Eng. Res. Des. 2006 84, 47.

204. Patience, D. B.; Rawlings, J. B. Particle-shape monitoring and control in crystallization processes. AIChE J. 2001 47, 2125.

205. Dharmayat, S.; Calderon De Anda, J.; Hammond, R. B.; Lai, X.; Roberts, K. J.; Wang, X. Z. Polymorphic transformation of l-glutamic acid monitored using combined on-line video microscopy and X-ray diffraction. J. Cryst. Growth 2006 294, 35.

206. Bernard-Michel, B.; Pons, M. N.; Vivier, H. Quantification, by image analysis, of effect of operational conditions on size and shape of precipitated barium sulphate. Chem. Eng. J. 2002 $87,135$.

207. Pons, M.-N.; Vivier, H.; Delcour, V.; Authelin, J.-R.; Pailleres-Hubert, L. Morphological analysis of pharmaceutical powders. Powder Technol. 2002 128, 276.

208. Faria, N.; Pons, M. N.; Feyo de Azevedo, S.; Rocha, F. A.; Vivier, H. Quantification of the morphology of sucrose crystals by image analysis. Powder Technol. 2003 133, 54.

209. Zhang, D.; Lu, G. Review of shape representation and description techniques. Pattern Recognition 2004 3\%, 1.

210. Yu, Z. Q.; Chow, P. S.; Tan, R. B. Quantification of particle morphology by boundary Fourier transform and generic Fourier transform. Chem. Eng. Sci. 2007 62, 3777.

211. Wang, X. Z.; Roberts, K. J.; Ma, C. Crystal growth measurement using 2D and 3D imaging and the perspectives for shape control. Chem. Eng. Sci. 2008 63, 1173.

212. Yang, G.; Kubota, N.; Sha, Z.; Louhi-Kultanen, M.; Wang, J. Crystal Shape Control by Manipulating Supersaturation in Batch Cooling Crystallization. Cryst. Growth Des. 2006 , 2799. 
213. Hermanto, M. W.; Chiu, M.-S.; Woo, X.-Y.; Braatz, R. D. Robust optimal control of polymorphic transformation in batch crystallization. AIChE J. 2007 53, 2643.

214. Fujiwara, M.; Nagy, Z. K.; Chew, J. W.; Braatz, R. D. First-principles and direct design approaches for the control of pharmaceutical crystallization. J. Process Control 2005 15, 493.

215. Larsen, P.; Patience, D.; Rawlings, J. Industrial crystallization process control. Control Systems Magazine, IEEE 2006 26, 70.

216. Yu, Z. Q.; Chew, J. W.; Tan, R. B. H. Recent advances in crystallization control. Chem. Eng. Res. Des. 2007 85, 893. 